\title{
Effects of Antibiotics on the Bacterial Community, Metabolic Functions and Antibiotic Resistance Genes in Mariculture Sediments during Enrichment Culturing
}

\author{
Meng-Qi Ye ${ }^{1}$, Guan-Jun Chen ${ }^{1,2}$ and Zong-Jun Du ${ }^{1,2, *}$ \\ 1 Marine College, Shandong University, Weihai 264209, Shandong, China; yemengqi@126.com (M.-Q.Y.); \\ guanjun@sdu.edu.cn (G.-J.C.) \\ 2 State Key Laboratory of Microbial Technology, Shandong University, Qingdao 266237, Shandong, China \\ * Correspondence: duzongjun@sdu.edu.cn
}

Received: 8 July 2020; Accepted: 11 August 2020; Published: 13 August 2020

\begin{abstract}
The effect of antibiotics on the diversity and functioning of indigenous microorganisms in the environment has attracted much attention. In this study, effects of exposure to six different antibiotics on the bacterial community, metabolic functions and antibiotic resistance genes (ARGs) in marine sediments during enrichment culturing were investigated. Classical culture-dependent method and high-throughput $16 \mathrm{~S}$ rRNA gene sequencing method were both applied. In the culture-dependent analysis, the obtained 1549 isolates belonged to four phyla (Proteobacteria, Firmicutes, Bacteroidetes and Actinobacteria) and 155 genera. Proteobacteria and Firmicutes were the dominant phyla. The diversity and abundance of obtained bacteria after antibiotic processing exhibited different degrees of decrease. Enrichment culturing for different time could also affect the bacterial community composition. Some genera of bacteria were not isolated in the control group, but they could be isolated in the antibiotic-treated groups. In high-throughput $16 \mathrm{~S}$ rRNA gene amplicon sequencing analyses, all the effective reads were clustered into 2822 OTUs at $97 \%$ similarity cutoff; they were annotated to 49 phyla, 103 class, 220 orders, 347 families, 624 genera and 1122 species. An alpha diversity analysis indicated that the community diversity and richness decreased under antibiotic exposure. The changes at the genus level were much more obvious. Only 48 genera of 129 genera were shared by all the samples. A total of 29 genera which were not detected in the initial control sample could be detected in at least one antibiotic-treated group. SIMPER analysis showed that OTU2543 and OTU1450 were the most common taxa to the dissimilarity of bacterial community between antibiotic-treated groups and the control group. OTU2034 and OUT2543 were the most contributive taxa to dissimilarity of groups incubating for different time. Metabolism was the predominant bacterial function. A total of $30 \mathrm{ARGs}$ were detected in the samples. This study mainly focused on the changes of microbiota under the selective pressure of antibiotics for different time and the results demonstrated that the antibiotic could affect the bacterial diversity and richness in the marine ecosystem.
\end{abstract}

Keywords: antibiotics; bacterial community; mariculture; culture-dependent method; high-throughput 16S rRNA gene sequencing; ARGs

\section{Introduction}

Antibiotics have been widely used in the human medical field, the aquaculture, the animal husbandry and agriculture, to prevent various infections and stimulate the growth of animals and plants since their discovery [1-5]. However, due to the increasing consumption of antibiotics globally 
in recent years [6], they also have caused extensive concern. They have been largely released into the environments by improper disposal or excretion by humans and animals [7-9], and not only cause chemical pollution, but also result in the dissemination of antibiotic resistance genes (ARGs) and epidemic of antibiotic resistant bacteria-or even multiresistant bacteria $[10,11]$. This is a serious and growing threat to ecosystems, human and animal health $[1,12,13]$. Moreover, the diversity and functioning of microorganisms will also be directly affected. Microorganisms play a crucial role in biogeochemical cycles, energy flows, food webs, contaminant bioremediation and organic matter mineralization $[14,15]$ and the community composition may cause functional variation [16]. Antibiotics may hinder the important ecosystem functions of microorganisms such as the natural degradation of organic contaminants [14], denitrification [17], nitrogen fixation [18], iron reduction [19], self-purification [20], which are very crucial processes [18]. Therefore, with the increasing use of antibiotics, the potential threat to environment cannot be neglected [21-23].

To date, some studies of microorganisms in the environment have focused on a few pathogenic bacteria based on culture-dependent methods [24-26]. More recently, many studies have explored the effects of antibiotics on the microbial communities in terrestrial ecosystems $[17,27,28]$, rivers, water-supply reservoirs ecosystems [15,29] and in the wastewater treatment process [30-32], based on the evolving gene-sequencing technology. Apart from these habitats, the marine environment is also a potential reservoir of antibiotics-especially the sediments. There have been many investigations on antibiotic exposure in sea; results show that antibiotic pollution is widespread in different regions, which was mainly due to terrestrial inputs and mariculture [33-36]. Under antibiotics selective pressure, the intrinsic bacterial communities may be affected in ways which will influence the exertion of their functions, and ultimately, may disturb mariculture, and even the marine ecosystem. In addition, different antibiotics have different selective capabilities [37].Hence, it is essential to study and compare the response of bacterial communities diversity of marine environment to different types of antibiotics [30]. In this study, we chose marine sediments as a research object to evaluate the effect of antibiotic exposure on bacterial communities during enrichment culturing-as well as metabolic functions and antibiotic resistance genes (ARGs). To achieve this goal, we conducted a laboratory experiment with surface-sediment samples collected from the inshore mariculture experimental area in Weihai, China. The samples were treated with six antibiotics (i.e., zinc bacitracin (ZIN), ciprofloxacin (CIP), ampicillin sodium (AMP), chloramphenicol (CHL), tylosin (TYL) and tetracycline (TET)), respectively for 30 days. The dynamic changes of bacterial communities were monitored based on not only classical culture-dependent methods, but also culture-independent microbial diversity analysis using high-throughput 16S rRNA gene sequencing. This study will help to evaluate the potential effect of antibiotics on the bacterial community of natural marine environment comprehensively. The study also enabled us to investigate if antibiotic could help to cultivate potential new species.

\section{Materials and Methods}

\subsection{Target Antibiotics}

In this study, six antibiotics belonging to six classes were selected as the targets. Information of the antibiotics is listed in detail in Table S1. Antibiotics were purchased from Sangon Biotic Co., Ltd. (Shanghai, China).

\subsection{Sample Collection and Experimental Design}

Sediment samples were collected in October 2016 from a sea cucumber farming experimental area at Rongcheng $\left(122^{\circ} 14^{\prime} 34^{\prime \prime}\right.$ E, $36^{\circ} 54^{\prime} 36^{\prime \prime}$ N) in Weihai, Shandong Province, China, where no antibiotics were applied during the cultivation of sea cucumber. The samples from five sites were mixed thoroughly in sterilized Whirl-Pak bags (NASCO, Modesto, CA, USA) and transferred with ice bags to the laboratory within $2 \mathrm{~h}$. 
Enrichment medium was prepared using seawater according to the following ingredients as our previous report [38]: $0.02 \%$ yeast extract, $0.02 \%$ tryptone, $0.02 \% \mathrm{MgSO}_{4} \cdot 7 \mathrm{H}_{2} \mathrm{O}, 0.1 \% \mathrm{NH}_{4} \mathrm{Cl}$, $0.1 \%$ EDTA, $0.125 \%$ sodium pyruvate, $0.2 \% \mathrm{CH}_{3} \mathrm{COONa}$. The $\mathrm{pH}$ of the medium was adjusted to 7.0 . After autoclaving, $2 \%(w / v) \mathrm{KH}_{2} \mathrm{PO}_{4}$ solution and $10 \%(w / v) \mathrm{NaHCO}_{3}$ solution $(10 \mathrm{~mL}$ per $\mathrm{L})$ were supplemented in the medium, which were autoclaved alone and filtered for sterilization, respectively. Six antibiotic-treated groups and a control group were set. The antibiotic-treated groups were treated with $50 \mathrm{mg} / \mathrm{L}$ of zinc bacitracin (ZIN), $30 \mathrm{mg} / \mathrm{L}$ of ciprofloxacin (CIP), $100 \mathrm{mg} / \mathrm{L}$ of ampicillin sodium (AMP), $30 \mathrm{mg} / \mathrm{L}$ of chloramphenicol (CHL), $50 \mathrm{mg} / \mathrm{L}$ of tylosin (TYL) and $30 \mathrm{mg} / \mathrm{L}$ of tetracycline (TET), respectively. Enrichment culture incubation under different antibiotic exposure was conducted in the dark to prevent possible photodegradation of antibiotics at $25^{\circ} \mathrm{C}$ for $0,5,12,21$ and 30 days in separate $500-\mathrm{mL}$ sealed glass bottles (filled with enrichment medium and $20 \mathrm{~g}$ of sediment sample). The cultures were carefully shaken by hand twice daily to ensure good homogenization of antibiotic and sediments. After incubation for 12 days, each antibiotic was re-added with same concentration as the previous treatment for constant high antibiotic exposure for comparison. The detailed design is shown in Table 1.

Samples from enrichment culture were taken at regular intervals $(0,5,12,21$ and 30 days of incubation). Each 50-mL sample was taken from a new bottle in triplicate. The sample was split into two parts-one for classical culture-dependent analysis, and the other-which was stored at $-80{ }^{\circ} \mathrm{C}$-was used to conduct Illumina high-throughput $16 \mathrm{~S}$ rRNA gene sequencing for analyzing culture-independent microbial community composition.

Table 1. Details of experimental design.

\begin{tabular}{|c|c|c|c|c|}
\hline Group & Designated as & Treatment & Concentration $(\mathrm{mg} / \mathrm{L})$ & Incubation Time (days) \\
\hline \multirow{5}{*}{ A } & $\mathrm{A} 0$ & Control & - & 0 \\
\hline & A1 & Control & - & 5 \\
\hline & A2 & Control & - & 12 \\
\hline & A3 & Control & - & 21 \\
\hline & A4 & Control & - & 30 \\
\hline \multirow{7}{*}{$\mathrm{P}$} & P0 & zinc bacitracin & 50 & 0 \\
\hline & P1 & zinc bacitracin & 50 & 5 \\
\hline & P2 & zinc bacitracin & 50 & 12 \\
\hline & P3 & zinc bacitracin & 50 & 21 \\
\hline & $\mathrm{P} 4$ & zinc bacitracin & 50 & 30 \\
\hline & JP3 & zinc bacitracin, re-added on Day 12 & $50+50$ & 21 \\
\hline & JP4 & zinc bacitracin, re-added on Day 12 & $50+50$ & 30 \\
\hline \multirow{7}{*}{ Q } & Q0 & ciprofloxacin & 30 & 0 \\
\hline & Q1 & ciprofloxacin & 30 & 5 \\
\hline & Q2 & ciprofloxacin & 30 & 12 \\
\hline & Q3 & ciprofloxacin & 30 & 21 \\
\hline & Q4 & ciprofloxacin & 30 & 30 \\
\hline & JQ3 & Ciprofloxacin, re-added on Day 12 & $30+30$ & 21 \\
\hline & JQ4 & Ciprofloxacin, re-added on Day 12 & $30+30$ & 30 \\
\hline \multirow{7}{*}{$\mathrm{R}$} & R0 & ampicillin sodium & 100 & 0 \\
\hline & R1 & ampicillin sodium & 100 & 5 \\
\hline & $\mathrm{R} 2$ & ampicillin sodium & 100 & 12 \\
\hline & R3 & ampicillin sodium & 100 & 21 \\
\hline & R4 & ampicillin sodium & 100 & 30 \\
\hline & JR3 & ampicillin sodium, re-added on Day 12 & $100+100$ & 21 \\
\hline & JR4 & ampicillin sodium, re-added on Day 12 & $100+100$ & 30 \\
\hline
\end{tabular}


Table 1. Cont.

\begin{tabular}{|c|c|c|c|c|}
\hline Group & Designated as & Treatment & Concentration $(\mathrm{mg} / \mathrm{L})$ & Incubation Time (days) \\
\hline \multirow{7}{*}{$\mathrm{S}$} & S0 & chloramphenicol & 30 & 0 \\
\hline & S1 & chloramphenicol & 30 & 5 \\
\hline & S2 & chloramphenicol & 30 & 12 \\
\hline & S3 & chloramphenicol & 30 & 21 \\
\hline & S4 & chloramphenicol & 30 & 30 \\
\hline & JS3 & chloramphenicol, re-added on Day 12 & $30+30$ & 21 \\
\hline & JS4 & chloramphenicol, re-added on Day 12 & $30+30$ & 30 \\
\hline \multirow{7}{*}{$\mathrm{T}$} & T0 & tylosin & 50 & 0 \\
\hline & $\mathrm{T} 1$ & tylosin & 50 & 5 \\
\hline & $\mathrm{T} 2$ & tylosin & 50 & 12 \\
\hline & T3 & tylosin & 50 & 21 \\
\hline & $\mathrm{T} 4$ & tylosin & 50 & 30 \\
\hline & JT3 & tylosin, re-added on Day 12 & $50+50$ & 21 \\
\hline & JT4 & tylosin, re-added on Day 12 & $50+50$ & 30 \\
\hline \multirow{7}{*}{$\mathrm{U}$} & U0 & tetracycline & 30 & 0 \\
\hline & U1 & tetracycline & 30 & 5 \\
\hline & $\mathrm{U} 2$ & tetracycline & 30 & 12 \\
\hline & U3 & tetracycline & 30 & 21 \\
\hline & $\mathrm{U} 4$ & tetracycline & 30 & 30 \\
\hline & JU3 & tetracycline, re-added on Day 12 & $30+30$ & 21 \\
\hline & JU4 & tetracycline, re-added on Day 12 & $30+30$ & 30 \\
\hline
\end{tabular}

\subsection{Bacterial Communities Based on Culture-Dependent Analysis}

For classical culture-dependent analysis, the samples were ten-fold serial-diluted with saline and the suspensions were spread on Marine agar 2216 (MA; Becton-Dickinson, NJ, USA) and cultivated routinely at $25{ }^{\circ} \mathrm{C}$. Each sample was spread on at least 30 plates. Without enriching in the liquid medium under antibiotic exposure, the samples of $\mathrm{P0}, \mathrm{Q} 0, \mathrm{R} 0, \mathrm{~S} 0, \mathrm{~T} 0$ and $\mathrm{U} 0$ were analyzed using MA with addition of the corresponding antibiotic. All the plates were cultivated at $25^{\circ} \mathrm{C}$ for 7 days, and colony counting was conducted after incubation for 3, 5 and 7 days. Only plates of 30-300 colonies were selected and the results were expressed in CFU/g [39]. All different colonies on the plate were picked, isolated and purified. The genomic DNA of each strain was extracted and purified using commercially available kit (Takara Bio, Dalian, China) and the 16S rRNA gene sequences were amplified by PCR with two universal primers $27 \mathrm{f}$ and $1492 \mathrm{r}$ [40]. Sequencing of the amplified $16 \mathrm{~S}$ rRNA gene was performed by Genewiz (Suzhou, China). The phylogenetic neighbors and 16S rRNA gene sequences similarity of the strains were obtained by using BLAST search (https://www.ncbi.nlm.nih.gov) and the EzTaxon-e server (http://www.ezbiocloud.net). The strains were maintained at $-80{ }^{\circ} \mathrm{C}$ in sterile $1 \%(w / v)$ saline supplemented with $15 \%(v / v)$ glycerol.

\subsection{Culture-Independent Bacterial Community Analysis Based on ILLUMINA HIGH-THROUGHPUT $16 S$} rRNA Gene Sequencing

Samples under different antibiotic treatments for different duration times were taken in triplicate and kept frozen at $-80{ }^{\circ} \mathrm{C}$ until culture-independent bacterial community analyses were performed. Triplicate samples collected from each bottle were mixed with equal qualities. The genomic DNA from samples were extracted and purified by commercial PowerSoil DNA isolation kit and Power Clean ProDNA clean-up kit (MoBio, San Diego, CA, USA). DNA quality was examined by $1 \%$ agarose gel electrophoresis. The V4-V5 hypervariable region of $16 \mathrm{~S}$ rRNA gene was amplified with the primer pair 515F and 907R (5'-GTGCCAGCMGCCGCGG-3' and 5'-CCGTCAATTCMTTTRAGTTT-3', 
respectively) [38]. Sequencing was performed by Majorbio Bio-Pharm Technology Co., Ltd. (Shanghai, China) using the Illumina MiSeq PE300 platform.

The raw sequences were processed using Trimmomatic and FLASH before analysis with the criteria as reported previously [41]. The post quality filtering reads after removing replication, chimera and singletons were then clustered into operational taxonomic units (OTUs) at $97 \%$ similarity threshold using the UPARSE-OTU algorithm, implemented as the cluster-OTUs command in USEARCH (version $7.0 \mathrm{http}: / /$ drive5.com/usearch/) [42]. The taxonomy of each 16S rRNA gene sequence was analyzed with QIIME [43] and the SILVA 119 [44] using a confidence threshold of 70\%. The subsequent analyses were performed on the free online platform of Majorbio I-Sanger Cloud Platform (www.i-sanger.com). The SIMPER analysis was conducted using vegan 2.5.6 in R 3.6.3.

\subsection{Prediction of Bacterial Functions and Analysis of Antibiotic Resistance Genes}

The functions of bacterial communities and antibiotic resistance genes in different samples were predicted using PICRUSt [45]. The analysis was performed on the free online platform of Majorbio I-Sanger Cloud Platform.

\section{Results and Discussion}

\subsection{The Changes in the Abundances and Diversity of Cultivable Bacteria under Different Antibiotic Pressure}

The abundance of cultivable bacteria in the samples under different antibiotic exposure for different incubation time were detected using colony-counting methods. The colony counting results were illustrated in Figure 1. Comparing the samples at time 0 (A0, P0, Q0, R0, S0, T0, U0), exposure to antibiotics could make the concentration of cultivable bacteria decrease in different degree, suggesting that the antibiotics had a negative effect on the microbial community. Especially the sample of S0, treated with chloramphenicol, the concentration of cultivable bacteria was lowest, i.e., $6.3 \times 10^{3} \mathrm{CFU} / \mathrm{g}$. This indicated the selective pressure of chloramphenicol was very high. After enrichment under antibiotic exposure for five days, the concentration of cultivable bacteria increased in all the antibiotic-treated groups, as well as in the control group. The number of colonies of sample Q1, R1 and U1 were even higher than that of A1. When the enrichment period was prolonged, the number of colonies in most of the samples decreased, which may be due to oxygen consumption, toxic metabolites accumulation and nutrient deficiencies, but the cultivable bacteria were still more than that of the samples at time 0 . Though antibiotics can inhibit growth of certain groups of bacteria, this situation may happen to create proper growth condition for several other kinds of bacteria, causing the colony number increase. In addition, when the antibiotics were re-added on Day 12 to consolidate the selective pressure, the cultivable bacteria in the samples collected on Day 21 were less than that of samples without re-adding antibiotics. This may be due to the concentration of antibiotic increasing and inhibiting more bacteria.

\subsection{Taxonomic Composition of Cultivable Bacteria}

Across all the samples, a total of 1549 isolates were picked and purified based on the phenotypic characteristic of colony color, size, shape, texture and growth pattern, etc. As shown in Table 2, the isolates obtained from control group were the most (Group A, 372 strains), followed by the zinc bacitracin-treated group (Group P, 254 strains), tylosin-treated group (Group T, 250 strains), tetracycline-treated group (Group U, 193 strains), chloramphenicol-treated group (Group S, 190 strains), ampicillin sodium-treated group (Group R, 156 strains) and ciprofloxacin-treated group (Group Q, 134 strains). The antibiotic treatment lowered the bacterial diversity. The strains obtained after enrichment culturing were more than that of the initial samples. The 16S rRNA gene of the strains were amplified, sequenced and aligned to be explicit about the taxonomic information. The number of phyla, genera and species that the isolates of each group distributed in was also shown in Table 2, comparing with the control group, the diversity of bacteria community after antibiotic processing exhibited different 
degrees of decrease. Exposing to the antibiotic for different time during enrichment, the diversity of bacteria community also changed obviously.

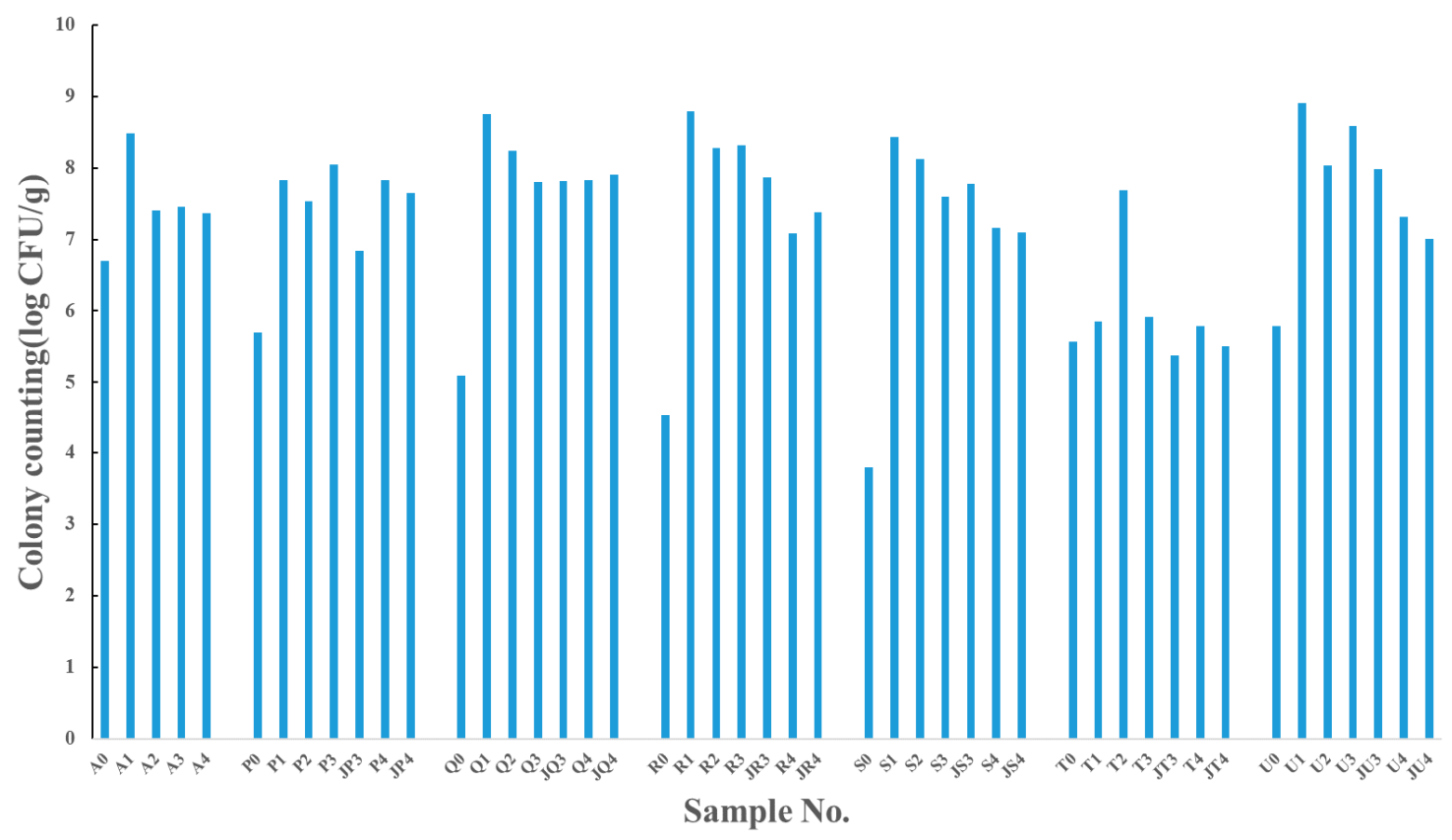

Figure 1. Cultured bacterial colonies counting under different antibiotic exposure.

Table 2. The number of isolates and taxonomic composition of culturable bacteria.

\begin{tabular}{|c|c|c|c|c|c|}
\hline \multirow{2}{*}{ Sample No. } & \multirow{2}{*}{ Enrichment Time (d) } & \multirow{2}{*}{ Number of Strains } & \multicolumn{3}{|c|}{ Taxonomic Composition } \\
\hline & & & Phyla & Genera & Species \\
\hline \multicolumn{2}{|c|}{ Control } & 372 & 4 & 76 & 145 \\
\hline A0 & 0 & 55 & 4 & 28 & 43 \\
\hline A1 & 5 & 58 & 4 & 18 & 32 \\
\hline A2 & 12 & 82 & 4 & 27 & 44 \\
\hline A3 & 21 & 98 & 4 & 35 & 63 \\
\hline A4 & 30 & 79 & 4 & 31 & 55 \\
\hline \multicolumn{2}{|c|}{ Exposure to zinc bacitracin $(\mathrm{P})$} & 254 & 4 & 67 & 137 \\
\hline P0 & 0 & 35 & 2 & 19 & 28 \\
\hline P1 & 5 & 16 & 4 & 12 & 14 \\
\hline P2 & 12 & 28 & 4 & 16 & 20 \\
\hline P3 & 21 & 50 & 4 & 24 & 36 \\
\hline JP3 & 21 & 44 & 4 & 22 & 35 \\
\hline P4 & 30 & 21 & 3 & 13 & 21 \\
\hline JP4 & 30 & 60 & 4 & 24 & 40 \\
\hline \multicolumn{2}{|c|}{ Exposure to ciprofloxacin $(\mathrm{Q})$} & 134 & 4 & 37 & 67 \\
\hline Q0 & 0 & 7 & 1 & 2 & 2 \\
\hline $\mathrm{Q} 1$ & 5 & 16 & 3 & 5 & 11 \\
\hline Q2 & 12 & 34 & 4 & 17 & 23 \\
\hline Q3 & 21 & 25 & 4 & 13 & 18 \\
\hline JQ3 & 21 & 24 & 4 & 12 & 18 \\
\hline Q4 & 30 & 14 & 4 & 9 & 10 \\
\hline JQ4 & 30 & 14 & 4 & 13 & 14 \\
\hline
\end{tabular}


Table 2. Cont.

\begin{tabular}{|c|c|c|c|c|c|}
\hline \multirow{2}{*}{ Sample No. } & \multirow{2}{*}{ Enrichment Time (d) } & \multirow{2}{*}{ Number of Strains } & \multicolumn{3}{|c|}{ Taxonomic Composition } \\
\hline & & & Phyla & Genera & Species \\
\hline \multicolumn{2}{|c|}{ Exposure to ampicillin sodium (R) } & 156 & 4 & 46 & 83 \\
\hline R0 & 0 & 15 & 3 & 12 & 13 \\
\hline R1 & 5 & 17 & 2 & 7 & 11 \\
\hline $\mathrm{R} 2$ & 12 & 31 & 4 & 11 & 19 \\
\hline $\mathrm{R} 3$ & 21 & 29 & 4 & 15 & 23 \\
\hline JR3 & 21 & 26 & 4 & 15 & 18 \\
\hline $\mathrm{R} 4$ & 30 & 19 & 3 & 14 & 17 \\
\hline $\mathrm{JR} 4$ & 30 & 19 & 4 & 13 & 18 \\
\hline \multicolumn{2}{|c|}{ Exposure to chloramphenicol (S) } & 190 & 4 & 48 & 85 \\
\hline SO & 0 & 5 & 2 & 3 & 3 \\
\hline S1 & 5 & 17 & 3 & 6 & 10 \\
\hline S2 & 12 & 39 & 4 & 17 & 27 \\
\hline S3 & 21 & 31 & 4 & 16 & 24 \\
\hline JS3 & 21 & 58 & 4 & 23 & 36 \\
\hline S4 & 30 & 20 & 4 & 15 & 17 \\
\hline JS4 & 30 & 20 & 3 & 12 & 15 \\
\hline \multicolumn{2}{|c|}{ Exposure to tylosin $(\mathrm{T})$} & 250 & 4 & 58 & 117 \\
\hline T0 & 0 & 5 & 1 & 2 & 34 \\
\hline $\mathrm{T} 1$ & 5 & 22 & 4 & 10 & 14 \\
\hline $\mathrm{T} 2$ & 12 & 31 & 2 & 11 & 23 \\
\hline $\mathrm{T} 3$ & 21 & 39 & 3 & 12 & 25 \\
\hline JT3 & 21 & 59 & 3 & 27 & 43 \\
\hline $\mathrm{T} 4$ & 30 & 44 & 4 & 18 & 26 \\
\hline JT4 & 30 & 50 & 4 & 21 & 37 \\
\hline \multicolumn{2}{|c|}{ Exposure to tetracycline (U) } & 193 & 4 & 51 & 93 \\
\hline U0 & 0 & 42 & 4 & 25 & 33 \\
\hline U1 & 5 & 19 & 4 & 8 & 9 \\
\hline $\mathrm{U} 2$ & 12 & 25 & 4 & 12 & 19 \\
\hline U3 & 21 & 30 & 3 & 12 & 17 \\
\hline JU3 & 21 & 39 & 4 & 19 & 28 \\
\hline $\mathrm{U} 4$ & 30 & 10 & 4 & 10 & 10 \\
\hline JU4 & 30 & 28 & 4 & 15 & 21 \\
\hline
\end{tabular}

The 16S rRNA gene identification results showed that the taxa were comprised of 4 prokaryotic phyla (Figure 2) and 155 genera (Figure 3). Further analyses of the community succession dynamics based on the differences at phylum level and genus level were also exhibited in Figures 2 and 3, respectively. It can be concluded that the different antibiotic exposure maintaining different time could affect the cultivable bacteria community composition greatly.

As shown in Figure 2, four prokaryotic phyla of bacteria were identified within all the samples, i.e., Actinobacteria, Bacteroidetes, Firmicutes and Proteobacteria. However, the abundance in the different samples varied from each other. On the whole, Proteobacteria and Firmicutes were the dominant phyla, accounted for $44.49 \%$ and $33.69 \%$, respectively in average in all the samples, followed by Bacteroidetes (14.16\%) and Actinobacteria (8.09\%). 


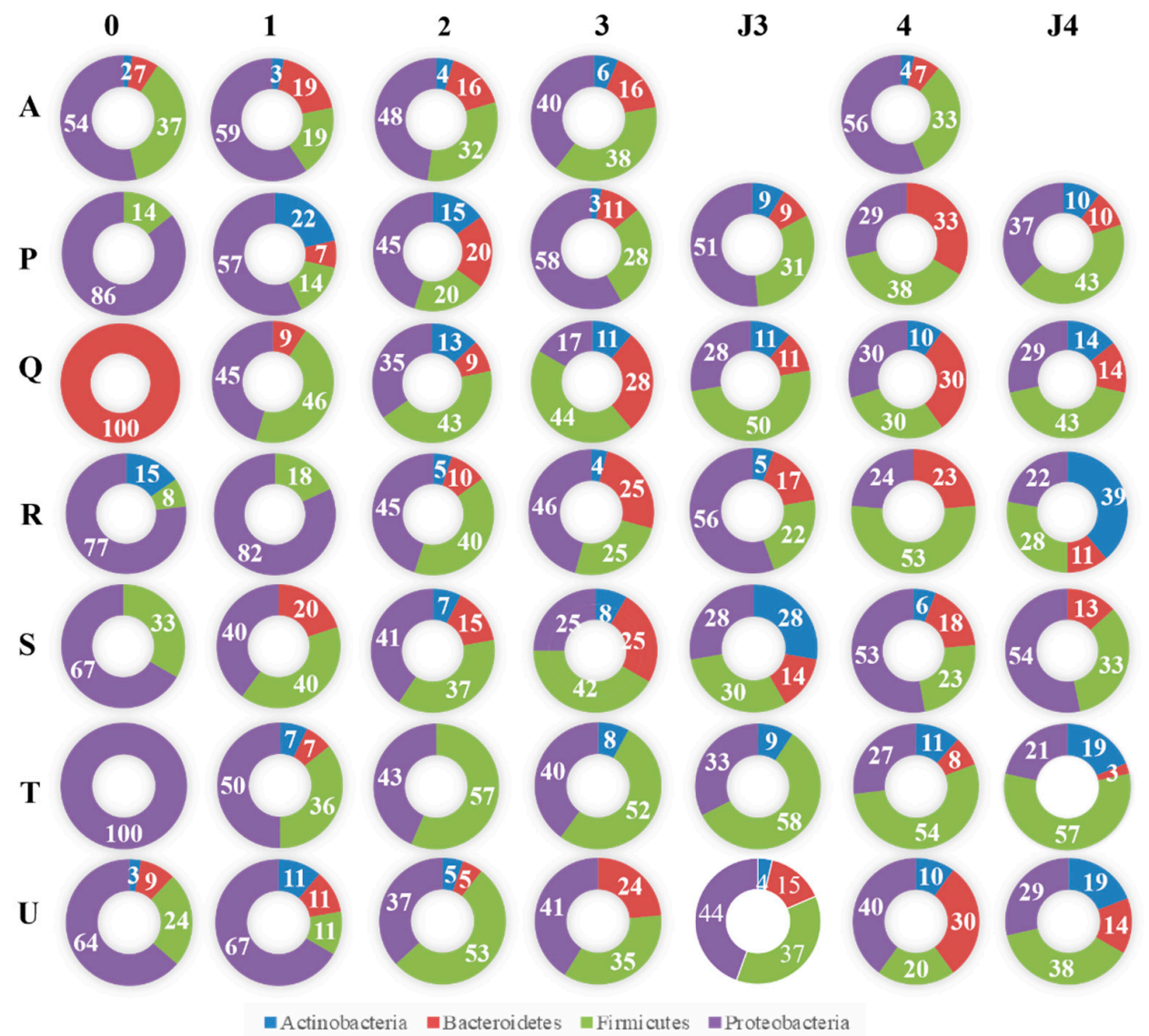

Figure 2. Culturable bacterial community compositions of all the samples at phylum level. Value represents the percentage $(\%)$. Values are rounded up to the nearest whole number.

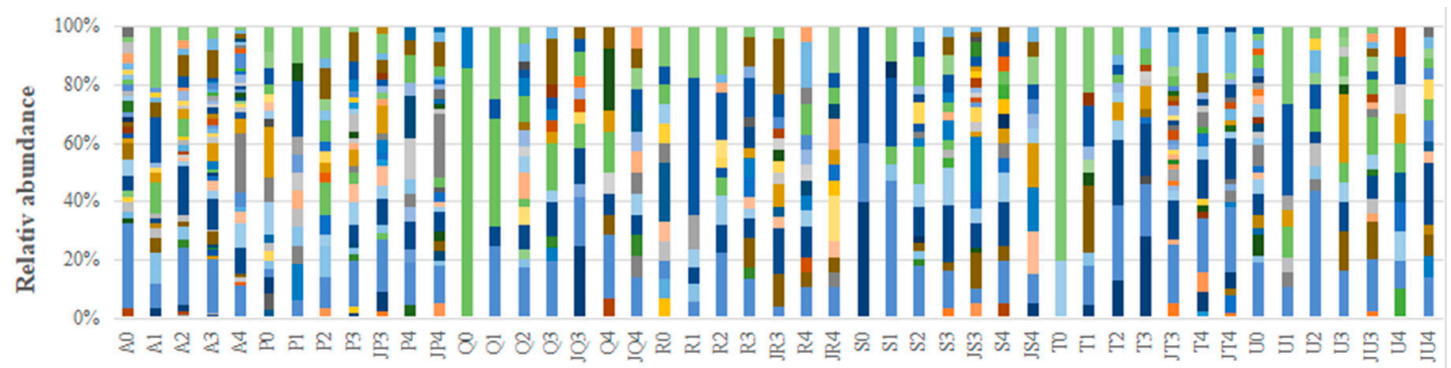

Samples

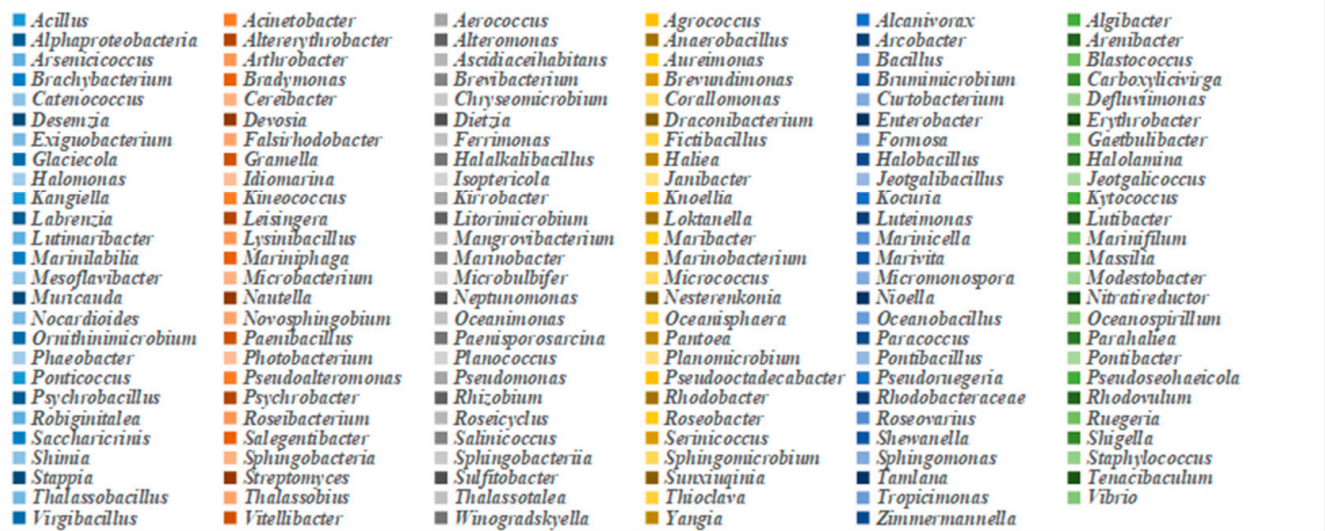

Figure 3. Culturable bacterial community compositions of all the samples at genus level. 
In the initial control sample A0, 43 strains were obtained after removing repetitions based on the 16S rRNA gene similarity, with the abundance of Proteobacteria, Firmicutes, Bacteroidetes and Actinobacteria being 53.49\%, 37.21\%, 6.98\% and 2.33\%, respectively (Figure 2). After enrichment culturing for 5, 12, 21 days, the proportions of Bacteroidetes went up, while the proportion of Proteobacteria decreased. However, the following 9 days of enrichment brought great change to the bacterial community again and on Day 30, in the sample of A4, the abundance of Proteobacteria, Firmicutes, Bacteroidetes and Actinobacteria were $56.36 \%, 32.73 \%, 7.27 \%$ and $3.64 \%$, respectively (Figure 2). These four phyla accounted for $51.33 \%, 31.72 \%, 12.96 \%$ and $4.00 \%$, respectively in average in the control group. At genus level, there were 28 genera in the sample A0, four phyla of Proteobacteria, Firmicutes, Bacteroidetes and Actinobacteria were comprised of 19 genera, 6 genera, 2 genera, 1 genus, respectively. When enriched for 5 days (A1), the number of genera decreased from 28 to 18 , but then the genera became diverse gradually and in the sample of A2, A3, A4, there were 27, 35 and 31 genera, respectively (Table 2, Figure 3). Thereinto, after enrichment for 5, 12, 21, 30 days, there were 12, 18, 26 and 21 genera that did not isolated from initial sample A0, being obtained, respectively, indicating that the enrichment for different time could affect the microbial community composition significantly.

Continuous exposure to different antibiotics all had great effect on the proportion of strains in different phylum and generate a significant shift in the sediment microbial community. In the zinc bacitracin-treated group $(\mathrm{P})$, before enrichment culturing, the strains belonged to Proteobacteria were overwhelming, accounting for $85.71 \%$, while the strains of Actinobacteria or Bacteroidetes were not isolated (Figure 2). This may be due to the zinc bacitracin inhibited the growth of strains of these two phyla directly. There were 19 genera in the sample P0 (Table 2, Figure 3). Although the diversity was poor comparing with the control sample A0, 12 of 19 genera were not isolated in the control sample. The selective pressure of zinc bacitracin changed the structure of cultivable bacterial community greatly. After enrichment culturing for a few days, the diversity increased obviously and strains of Actinobacteria and Bacteroidetes were isolated and there were 6, 7, 13 12,9 and 16 genera in the sample $\mathrm{P} 1, \mathrm{P} 2, \mathrm{P} 3, \mathrm{JP} 3, \mathrm{P} 4$ and JP4, respectively being newly isolated comparing with sample A0 and P0 (Figure 3). The second addition of zinc bacitracin could change the structure of cultivable bacteria further and promote the isolation of more genera. In comparison with the sample P0 which did not experience enrichment culturing under the pressure of zinc bacitracin, there were altogether 48 genera being isolated after enrichment culturing.

The inhibitory effect of ciprofloxacin (Group Q) was so strong that only seven strains of Bacteroidetes, including two genera of Marinifilum and Saccharicrinis, were isolated in the sample Q0 (Table 2, Figure 3). Interestingly, these two genera were not isolated in the control sample A0. Under the selective pressure of ciprofloxacin, some strains could grow well instead. The number and diversity of strains were both enhanced after enrichment and the strains of four phyla were isolated. After incubating under ciprofloxacin pressure for 5, 12, 21, 30 days, 5, 17, 13 and 9 genera were isolated, respectively. Thereinto, 1, 9, 6 and 4 genera were newly isolated, respectively comparing with sample $\mathrm{A} 0$ and $\mathrm{Q} 0$. In the sample of JQ3 and JQ4, 12 and 13 genera were isolated, which were more abundant than the corresponding samples of Q3 and Q4, suggesting that the second addition of ciprofloxacin could help to increase the diversity of bacterial community (Table 2).

The similar situation also appeared in the tylosin-treated group (Group T). Only five strains of Proteobacteria were isolated in the sample T0, which included two genera of Vibrio and Halomonas (Table 2, Figure 3). However, after incubation for 30 days, the strains of the other three phyla were also isolated, and the strains were distributed in 18 genera (Table 2, Figure 3). The second addition of antibiotics also could make the bacterial community structure be different, even the bacterial diversity was much better.

Upon addition of ampicillin sodium (Group R), 15 strains distributing in three phyla and 12 genera were obtained after removing repetition in the sample $\mathrm{R} 0$ and the phylum of Bacteroidetes was not isolated (Table 2, Figure 2). When incubating for five days, the diversity of the cultivable strains became worse and isolates distributed in two phyla (Proteobacteria and Firmicutes) and seven genera (Table 2, 
Figures 2 and 3). This may be due to that some sensitive strains were directly inhibited by ampicillin sodium during the initial five days and the other groups depending on the interaction effect had not multiplied. Incubating for longer time, the diversity of the ampicillin sodium-treated samples became better. In the samples of R2, R3, JR3, R4 and JR4, 11, 15, 15, 14 and 13 genera were isolated, respectively (Table 2, Figure 3). It was worth noting that, in the sample of R4, the phylum of Actinobacteria was not isolated again, but the second addition of ampicillin sodium made the bacterial community of sample JR4 more different, in which phylum of Actinobacteria including six genera (Microbacterium, Brevibacterium, Janibacter, Knoellia, Kocuria, Microbacterium) were isolated (Figures 2 and 3).

Under the selective pressure of chloramphenicol (Group S), most of the species were inhibited and only genera of Arcobacter and Shewanella belonging to Proteobacteria, as well as genus of Bacillus belonging to Firmicutes were isolated in the sample S0 (Figure 3). After enrichment culturing for different time, the number and diversity of the cultivable strains both increased in some degree. Especially when incubating for 21 days, 16 genera belonging to 4 phyla were isolated, of which 11 genera were not isolated in the control sample A0 (Table 2, Figure 3). More notably, there were 23 genera being isolated in the sample JS3 upon second addition of chloramphenicol, in which 16 genera were not isolated in A0, and 12 genera were not isolated in sample S3 (Table 2, Figure 3). This was very convincing that the second addition of antibiotic providing continuous selective pressure could affect even promote the structure and diversity of cultivable bacteria.

Within the Group $U$, the addition of tetracycline did not bring such adverse inhibitory effect to the cultivable strains as the other antibiotics, so there were 25 genera being isolated in the sample U0 (Table 2, Figure 3). Be clearly different from the other groups, enrichment culturing actually made the diversity of tetracycline-treated samples decreased in some extent. In the samples of U1, U2, U3, JU3, $\mathrm{U} 4$ and JU4, only 8, 12, 12, 19, 10 and 15 genera were isolated, respectively. The second addition of tetracycline also could improve the diversity of the cultivable bacteria. Under the selective pressure of tetracycline for different time, the composition of the cultivable bacteria community changed and there were also some genera being isolated just after enrichment comparing with sample U0.

On the whole, at the genus level, exerting antibiotic exposure for different duration could change the bacterial diversity and accelerate microbial succession greatly. The diversity and abundance of different genera in the control group and antibiotic-treated groups were illustrated clearly in Figure 3. In the control sample (A0), strains of 28 genera were found. The bacterial communities changed with the extension of incubation time, and strains of another 48 genera were isolated after enrichment culturing. In comparison with the control group, the number of genera declined in all the antibiotic-treated groups, but the bacterial community composition changed dramatically. Some genera of bacteria were inhibited and not isolated, while up to 79 genera of bacteria appeared and were isolated, which were not isolated in the control group. Among these 79 genera, 33 genera could be isolated in the zinc bacitracin-treated group; 12 genera could be isolated in the ciprofloxacin-treated group; 15 genera could be isolated in ampicillin sodium-treated group; 13 genera could be isolated in the chloramphenicol-treated group; 29 genera could be isolated in tylosin-treated group and 13 genera could be isolated in tetracycline-treated group. Two genera (Microbacterium and Staphylococcus) were found in all the antibiotic-treated groups (Figure 3). The same genera exhibited different abundance under different antibiotic stress. The different antibiotics inhibited diverse microbial groups, and the natural community interaction was disturbed, which may provide proper conditions for the growth of some bacteria to facilitate effective culture and isolation. In order to compare antibiotic-treated groups with control group more clearly, we combined the samples of different incubation time under the same treatment group, and the heat map of species abundance and diversity at the level of genus was charted in Figure 4. The diversity and abundance of bacteria were highest in the control group. The antibiotic selective pressure did change the number and diversity of cultivable bacteria greatly. 

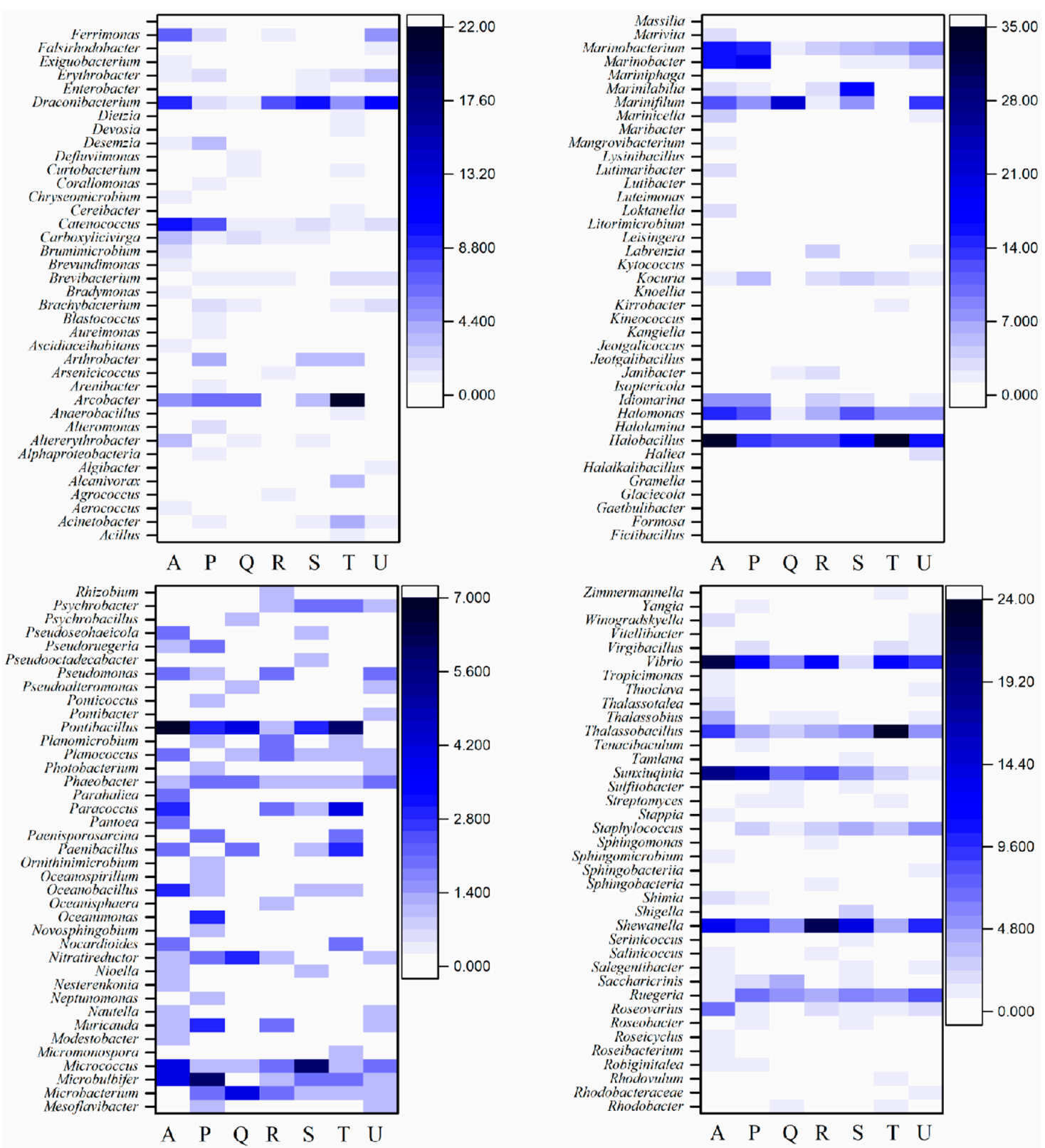

Figure 4. Culturable bacterial community compositions of different groups at genus level.

Although the number and diversity of the isolated strains under the antibiotic exposure decreased in some degree comparing with the control group, the community structure changed greatly, and there were still so many bacteria could survive even thrive, indicating a large number of bacteria were becoming resistant to antibiotics. Moreover, as the antibiotic treatment time lengthened, more bacteria could be isolated. This may be due to the antibiotic resistance genes (ARGs) propagation caused by the changes of environmental conditions and microbial community or long-term exposure to antibiotic $[30,46]$.

In addition, it was worth noting that there were some isolates whose $16 \mathrm{~S}$ rRNA gene sequences similarities with the identified strains were less than $98.5 \%$, suggesting that these isolates may be potential novel bacterial species. This provides a new potential method for cultivation and isolation of novel bacteria. 


\subsection{Microbial Community Characteristics Based on the High-Throughput 16S rRNA Gene Amplicon Sequencing}

\subsubsection{Diversity and Richness of Microbial Community}

After filtering, trimming and chimera removing, a total of 2,478,201 effective reads for all the samples were obtained. The number of effective reads per sample ranged from 50,636 to 73,795, and they were clustered into 2822 OTUs at $97 \%$ similarity cutoff. The coverage, ranging from $98.7 \%$ to $99.4 \%$, indicating that the OTUs of each sample were well captured and the sequencing depth was sufficient. Based on the alpha diversity indices, the abundance and diversity of the microbial community in different groups were different from each other during 30 days of incubation (Table 3). The sample $\mathrm{A} 0$, which was the raw sample without antibiotic exposure and enrichment culturing, had the highest indices of Sobs, Chao and Shannon, indicating the highest community richness and diversity of A0. However, interestingly, the Simpson index of A0 was the lowest, which suggested that the species evenness or equitability of A0 was low. Incubating for longer time, the species richness of samples without antibiotic (A1, A2, A3, A4) decreased, while the species evenness increased. The Sobs, Shannon, $\mathrm{ACE}$ and Chao indices of samples under antibiotic exposure were significantly lower than those of A0 sample, indicating the community diversity and richness decreased under antibiotic exposure. For each treatment, extending the time of incubation all had some effect on these indices. Comparing the samples of Day 5 under antibiotic treatments (P1, Q1, R1, S1, T1, U1) with the control one (A1), the selective pressure of zinc bacitracin, ciprofloxacin, ampicillin sodium and chloramphenicol made the microbial diversity lower. Instead, tylosin and tetracycline improved the microbial diversity. When incubating for 12 days, the microbial diversity of all the samples increased to different extent comparing with those of Day 5. Continuing to extend the enrichment time, different changes took place under the different treatments. For each antibiotic treatment, second addition of antibiotic on Day 12 made the microbial community differ from those of the samples without re-adding the antibiotic. Comparing the data on Day 30, re-addition of zinc bacitracin, ampicillin sodium, chloramphenicol and tylosin could further lowed the microbial diversity, but re-addition of ciprofloxacin and tetracycline raised the diversity, instead.

Table 3. Alpha diversity indices of microbial phylotypes.

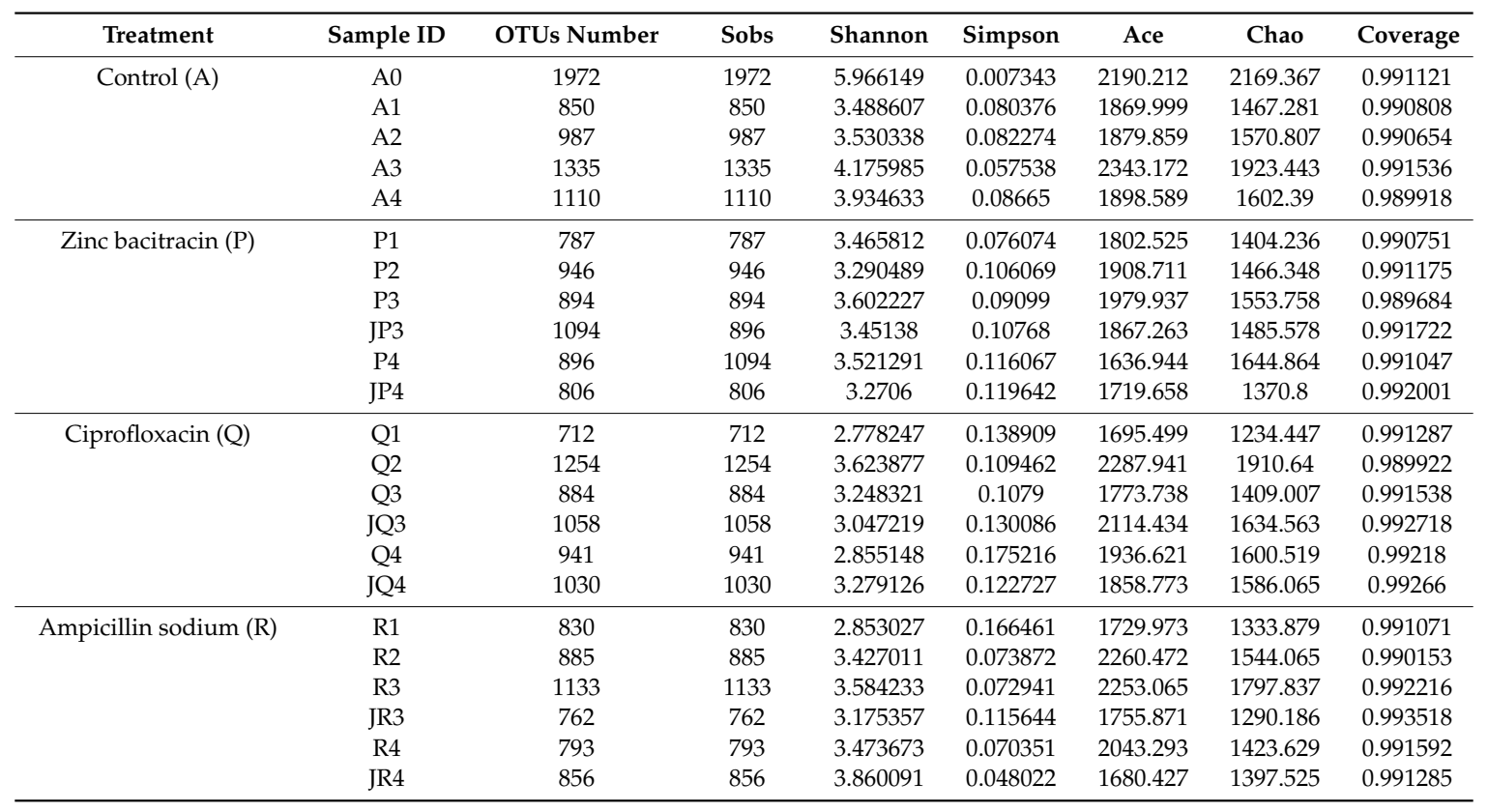


Table 3. Cont.

\begin{tabular}{|c|c|c|c|c|c|c|c|c|}
\hline Treatment & Sample ID & OTUs Number & Sobs & Shannon & Simpson & Ace & Chao & Coverage \\
\hline \multirow[t]{5}{*}{ Chloramphenicol (S) } & S1 & 705 & 705 & 2.904588 & 0.129851 & 2197.763 & 1459.063 & 0.992152 \\
\hline & $\mathrm{S} 2$ & 982 & 982 & 3.618378 & 0.069773 & 1780.536 & 1528.923 & 0.992284 \\
\hline & S3 & 776 & 776 & 3.318475 & 0.099976 & 1577.62 & 1306.472 & 0.992443 \\
\hline & $\mathrm{S} 4$ & 1056 & 1056 & 3.737706 & 0.074712 & 1965.486 & 1692 & 0.99179 \\
\hline & JS4 & 709 & 709 & 3.23018 & 0.09656 & 1629.057 & 1183 & 0.992223 \\
\hline \multirow{4}{*}{ Tylosin (T) } & $\mathrm{T} 3$ & 700 & 700 & 2.380128 & 0.187307 & 1584.71 & 1165.124 & 0.9944 \\
\hline & JT3 & 656 & 656 & 2.568263 & 0.231294 & 1831.557 & 1269.085 & 0.992333 \\
\hline & $\mathrm{T} 4$ & 1138 & 1138 & 3.452094 & 0.077866 & 1690.838 & 1601.896 & 0.992349 \\
\hline & JT4 & 706 & 706 & 2.336018 & 0.204594 & 2000.348 & 1395.545 & 0.99165 \\
\hline \multirow[t]{2}{*}{ Tetracycline (U) } & $\mathrm{U} 1$ & 1021 & 1021 & 3.614356 & 0.069014 & 2029.165 & 1648.687 & 0.988888 \\
\hline & JU4 & 811 & 811 & 2.829413 & 0.137191 & 1585.21 & 1257.515 & 0.993866 \\
\hline
\end{tabular}

The shared and unique OTUs between control group and the antibiotic-treated groups were calculated. Combining the samples of the same treatment group as a whole, the number of shared OTUs between control group (A) and group $\mathrm{P}$, group $\mathrm{Q}$, group $\mathrm{R}$, group $\mathrm{S}$, group $\mathrm{T}$ and group $\mathrm{U}$, were 1748, 1804, 1720, 1700, 1872 and 1790, respectively. As shown in Figure 5, the number of shared OTUs of all the seven groups was 886. The unique OTUs in the group A, group P, group Q, group R, group S, group $\mathrm{T}$ and group $\mathrm{U}$ were 48, 7, 10, 18, 13, 27 and 4, respectively. Each group induced the occurrence of some unique OTUs, indicating the change of the microbial community structure under antibiotic selective pressure. For the same treatment, the different exposure time to antibiotic also affected the shared OTUs and unique OTUs. These results are shown in Figure S1. The samples exposing to antibiotics for different time all had their specific OTUs, suggesting that after enrichment culturing for different time, the microbial community composition would change, and some new bacterial groups could be detected. Detailed analysis of species composition was conducted in the following section.

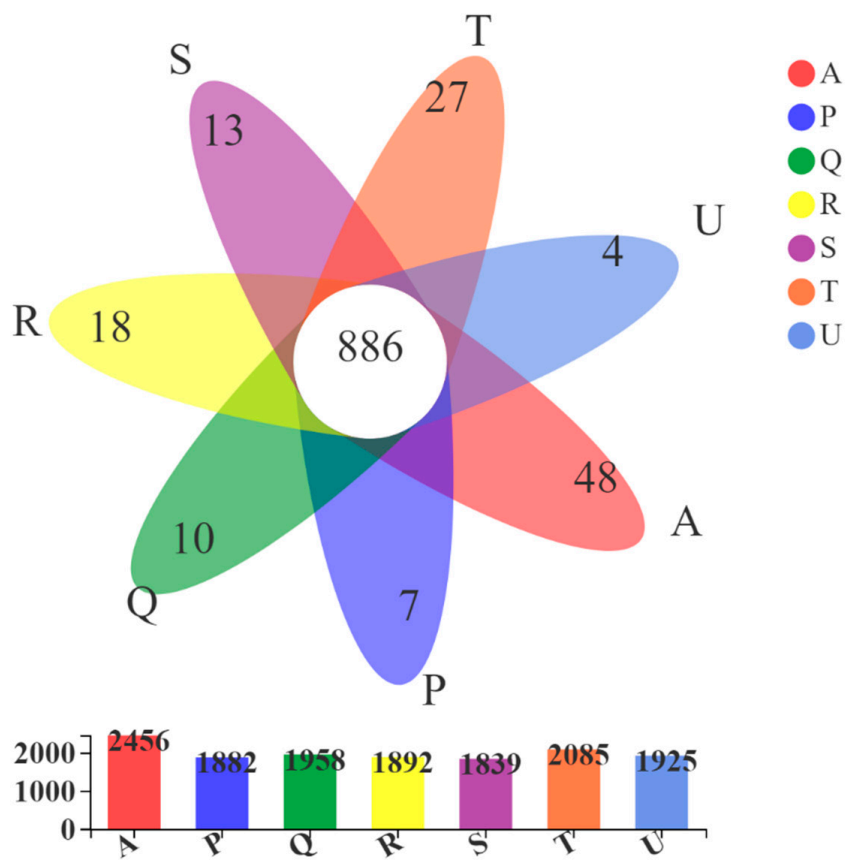

Figure 5. Number of unique and shared operational taxonomic units (OTUs) in the different groups. 


\subsubsection{Impact of Antibiotic on the Microbial Community at Phylum Level}

The 2822 OTUs in all the samples were annotated to 49 phyla, 103 class, 220 orders, 347 families, 624 genera and 1122 species. A total of 221 OTUs were not classified. The composition and relative abundance of different phyla in all samples is shown in Figure 6. Notably, the most dominant phylum in terms of average abundance in all the samples was Proteobacteria (43.31\%). Within Proteobacteria, Deltaproteobacteria was the most abundant class in all samples, followed by Gammaproteobacteria. The average abundance of Deltaproteobacteria in the control group (A) and P, Q, R, S, T and U groups were $28.75 \%, 42.40 \%, 34.47 \%, 20.18 \%, 28.08 \%, 29.99 \%$ and $39.91 \%$, respectively. The average abundance of Gammaproteobacteria were $10.67 \%, 6.55 \%, 5.00 \%, 16.17 \%, 14.53 \%, 7.39 \%$ and $6.83 \%$, respectively (Figure S2). Except for the most abundant Proteobacteria, phyla of Bacteroidetes $(17.30 \%)$, Firmicutes (15.82\%), Fusobacteria (11.45\%) and Spirochetae $(4.72 \%)$ were also abundant. In the sludge treatment wetlands, which are recognized as reservoirs of antibiotics, the phyla of Proteobacteria, Bacteroidetes and Firmicutes were also most abundant [47]. The other phyla were rare and accounted for less than $1.0 \%$. The abundance of the main phyla in different antibiotic treatment groups varied apparently comparing with the control group (Figure 7 and Table S2). Take the most abundant phylum Proteobacteria for example, the average abundance in the control group (A) and P, Q, R, S, T and U groups were $43.01 \%, 50.24 \%, 40.61 \%, 37.55 \%, 43.63 \%, 40.22 \%$ and $47.85 \%$, respectively (Figure 7 and Table S2).

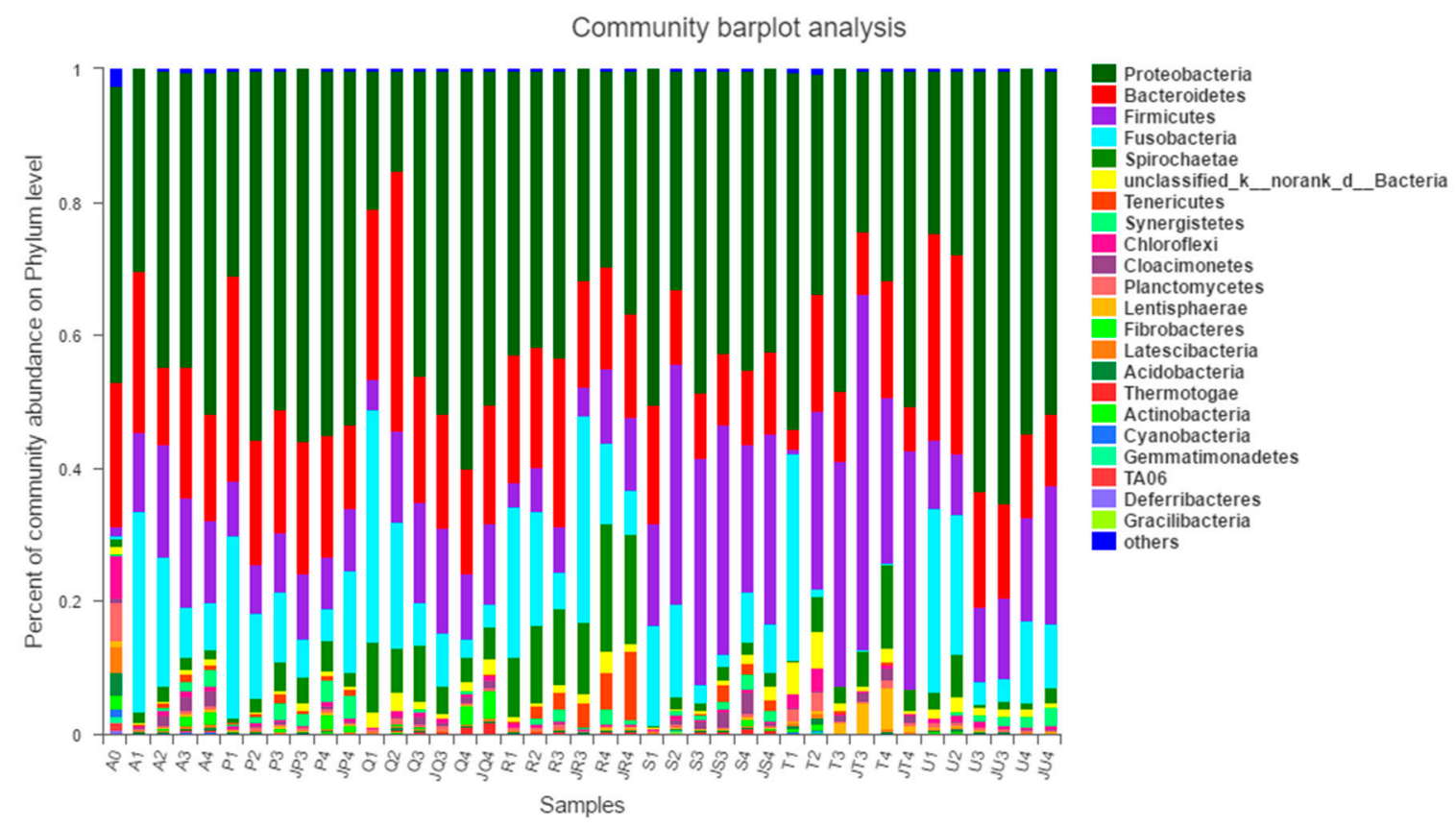

Figure 6. Composition and relative abundance of different phyla in all samples. A-control group; $\mathrm{P}$-zinc bacitracin-treated group; $\mathrm{Q}$ - ciprofloxacin-treated group; $\mathrm{R}$-ampicillin sodium-treated group; S-chloramphenicol-treated group; T-tylosin-treated group; U-tetracycline-treated group. The sample ID is composed of group abbreviation and number representing enrichment time. Key: 0—without enrichment; 1 - enrich for 5 days; 2-enrich for 12 days; 3-enrich for 21 days; 4 - enrich for 30 days. "Others" indicates the sum of the relative percentages of phyla with their maximum abundance lower than $0.5 \%$ in any sample. 


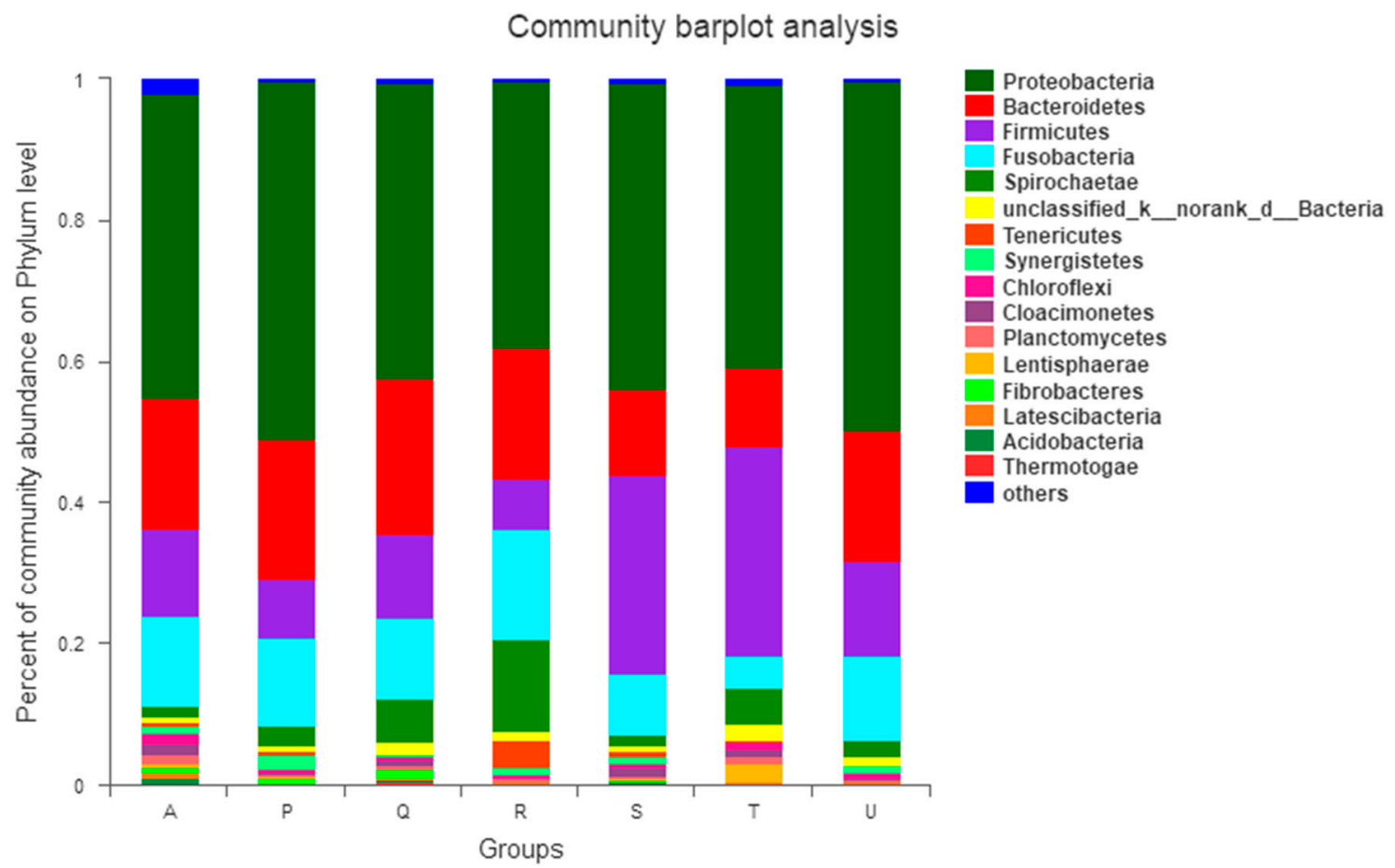

Figure 7. Comparison of the composition and relative abundance of different phyla of seven groups. The Group ID explanation is the same as that in the caption of Figure 6. "Others" indicates the sum of the relative percentages of phyla with their maximum abundance lower than $0.5 \%$ in any sample.

The diversity of bacterial community in the control sample A0 was the highest, in which 1972 OTUs were clustered and annotated to 48 phyla, 97 class, 203 orders, 303 families, 474 genera and 833 species. The main phyla of sample A0 was Proteobacteria (44.58\%), Bacteroidetes (21.75\%), Chloroflexi $(6.54 \%)$, Planctomycetes (5.8\%), Latescibacteria (3.83\%), Acidobacteria (3.47\%), Actinobacteria $(2 \%)$, Firmicutes $(1.24 \%)$, Spirochetae $(1.16 \%)$, Cyanobacteria (1.14\%), Gemmatimonadetes $(1.04 \%)$. The other phyla were rare and accounted for less than $1.0 \%$ (Figure 6). After enrichment culturing for different time, the diversity of microbial community of control sample decreased, and the abundance of different phylum changed greatly. The abundance of Firmicutes and Fusobacteria increased obviously after enrichment culturing, while Chloroflexi, Planctomycetes, Latescibacteria, Acidobacteria and Actinobacteria decreased. After enrichment culturing for 30 days, the most abundant phyla in the sample A4 were Proteobacteria (51.34\%), Bacteroidetes (16.02\%), Firmicutes (12.17\%) and Fusobacteria (7.18\%). The other phyla were extremely rare and the species evenness was poor (Figure 6). This may be due to that the decreasing nutrients or oxygen and increasing inhibitor occurred in the enrichment course affected some bacteria groups and their interactions.

In the zinc bacitracin-treated group (Group P), after enrichment culturing for 5 days under the pressure of zinc bacitracin, the number of annotated phyla decreased to 38 (Figure 6). The great change of abundance occurred in the phyla of Proteobacteria, Bacteroidetes, Firmicutes, Fusobacteria, Chloroflexi and Planctomycetes. After 30 days, the most abundant phyla were also Proteobacteria, Bacteroidetes, Fusobacteria and Firmicutes (Figure 6).

Under the pressure of ciprofloxacin (Group Q), the abundance of Spirochetae increased from $1.16 \%$ to $3.77 \%-10.54 \%$. After incubation for 5 days, the abundance of phylum Fusobacteria increased greatly, from $0.49 \%$ to $34.79 \%$, but then it decreased gradually and accounted for $2.77 \%$ and $3.37 \%$ in sample Q4 and JQ4, respectively, which indicated that the second addition of ciprofloxacin helped to further improve the abundance of Fusobacteria. Some phyla were not detected after ciprofloxacin treatment, including Armatimonadetes, Chlamydiae, Caldiserica, Nitrospirae (Figure 6). 
Under the selective pressure of ampicillin sodium (Group R), the abundance of Spirochetae also increased, from $1.16 \%$ to $8.77 \%-19.05 \%$, making it became another dominant phylum apart from Proteobacteria, Bacteroidetes, Firmicutes and Fusobacteria. The phyla of Lentisphaerae, Cyanobacteria, Fibrobacteres, Deferribacteres, Aminicenantes were not detected after the selective pressure of ampicillin sodium (Figure 6).

In the chloramphenicol-treated group (Group S), the most dominant phylum was Proteobacteria $(33.03 \%-50.43 \%)$, followed by Firmicutes $(15.47 \%-36.07 \%)$, Bacteroidetes $(9.81 \%-17.67 \%)$ and Fusobacteria $(1.91 \%-15.02 \%)$. The abundance of these four phyla accounted for $85.85 \%-98.59 \%$ (Figure 6). However, in fact, comparing with sample A0, the abundance of Bacteroidetes decreased under the pressure of chloramphenicol, same situation also occurred on the phyla of Acidobacteria, Latescibacteria, Actinobacteria, Elusimicrobia, Aminicenantes, Verrucomicrobia and Hydrogenedentes. The second addition of chloramphenicol also could make some difference.

In the tylosin-treated group (Group T), after treatment for 5 days, the abundance of Proteobacteria and Fusobacteria rose rapidly to $53.86 \%$ and $30.93 \%$, respectively. However, prolonging the incubation time, their abundance decreased, especially Fusobacteria, whose abundance decreased to $1.15 \%$ at Day 12 and $0.01 \%$ at Day 30 . This phenomenon showed that applying the stress of tylosin for a short time would help the competitive advantage of Fusobacteria. Instead, long-term pressure would inhibit the growth of Fusobacteria. The abundance of Firmicutes decreased at first and then rose. It decreased from $1.24 \%$ to $0.73 \%$ under tylosin pressure for 5 days, then rose to $26.73 \%-53.41 \%$ during the next 25 days. The changes of Fusobacteria and Firmicutes went through the opposite process. Beyond this, the abundance of Acidobacteria, Actinobacteria, Latescibacteria, Cyanobacteria, Gemmatimonadetes, Deferribacteres, Aminicenantes, Chlorobi and Verrucomicrobia decreased significantly (Figure 6). In the study of bacterial community of estuary reservoir, $\mathrm{Xu}$ et al. also found that tylosin had significant effect on variations of bacterial community composition [48].

When adding tetracycline (Group U), the abundance and diversity of bacterial community also changed greatly. The abundance of Bacteroidetes decreased to $10.62 \%-12.50 \%$ after 30 days of pressure. While the abundance of Fusobacteria, Proteobacteria and Firmicutes increased to $9.60 \%-12.46 \%$, $51.76 \%-54.81 \%$ and $15.44 \%-20.74 \%$, respectively. These four phyla were the dominant after tetracycline treatment for 30 days. Zhang et al. also found that the relative abundance of Proteobacteria increased after tetracycline exposure [49]. Some phyla decreased significantly under the selective pressure of tetracycline, including Chloroflexi, Planctomycetes, Acidobacteria, Latescibacteria and Actinobacteria (Figure 6).

In summary, the microbial community dynamics showed that the addition of antibiotics caused a great change in the abundance of main phyla in the samples.

\subsubsection{Impact of Antibiotic on the Microbial Community at Genus Level}

624 genera were assigned in all the samples. Combining the genera whose abundance were lower than $0.5 \%$ in all the samples, a total of 129 genera were obtained, among which 48 genera were shared by all the samples in different abundance. However, it is noticeable that the unclassified genera in the samples accounted for a very high percentage $(4.64 \%-53.32 \%)$. These 129 genera in all the samples were compared in Figure 8. 

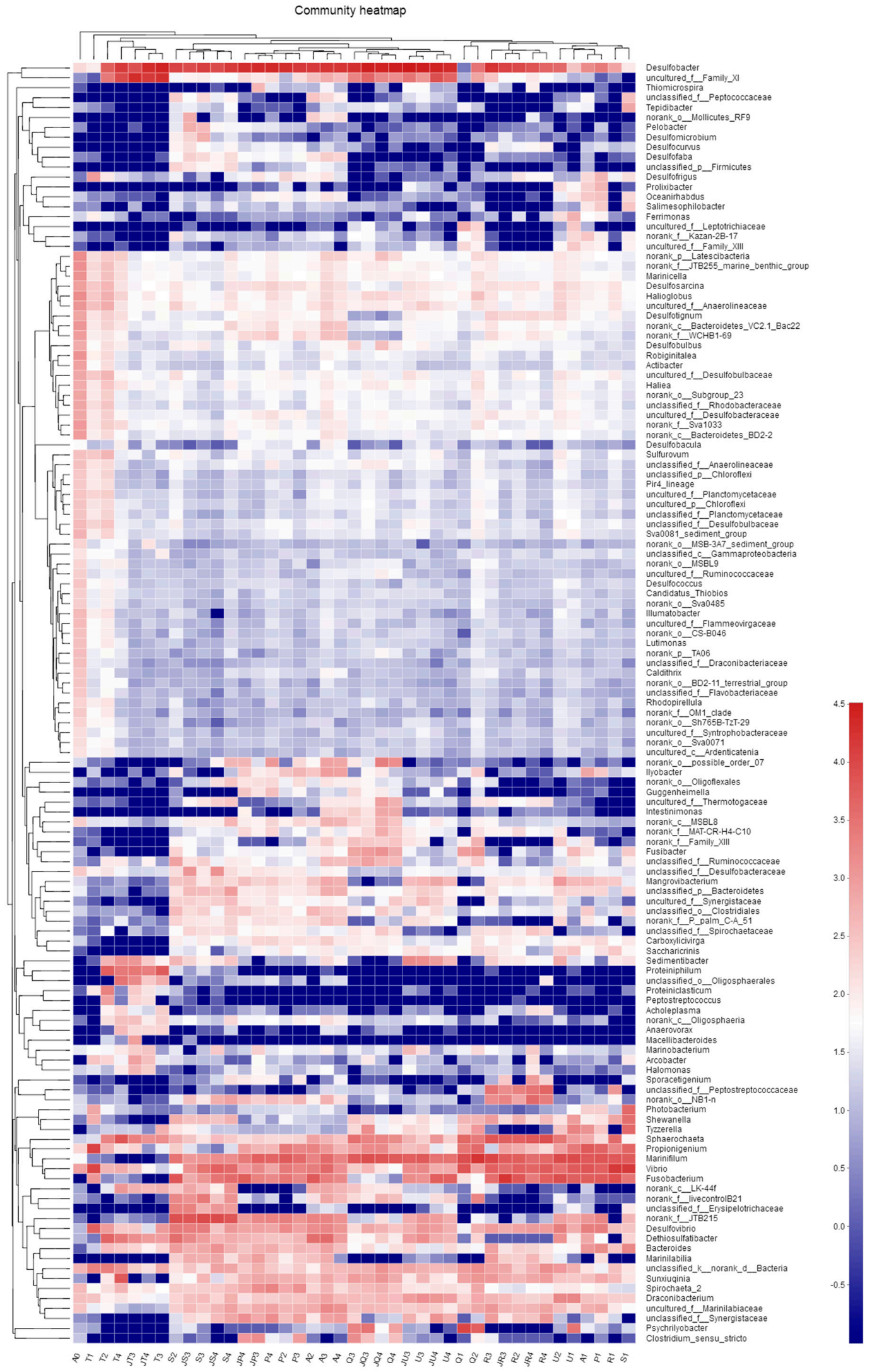

Figure 8. Abundant genera (abundance $>0.5 \%$ ) for each sample (a total of 129 genera for all samples). Both genus and the samples were ordered by the attribution order of cluster analysis. 
In addition, the dominant genera with an average abundance higher than $1 \%$ in the samples of the same treatment group were summarized in Table S3. The dominant genera in different treatment groups had both similarities and differences. There were some genera dominant in more than one group (Table S3). Further than that, there were 3 genera with an average abundance over $1 \%$ in all the groups, i.e., Desulfobacter (17.07\%-37.40\%), Vibrio (3.49\%-14.29\%) and Propionigenium $(1.37 \%-5.03 \%)$ (Table S3). Some genera were dominant in the control group and the addition of antibiotic caused a great decrease in the abundance, for example, genera Latescibacteria accounted for $3.74 \%$ in the control sample A0, but the addition of all the six antibiotics could decreased its abundance to lower than $1 \%$. Some genera with low abundance in the control group became the abundant genera in the antibiotic-treated groups. An unclassified genus of family Synergistaceae accounted for $0.01 \%$ in the control sample A0 and $0.79 \%$ in average in the control group. When adding zinc bacitracin (Group P), the abundance of this genus increased gradually and reached the maximum in the sample JP4 (3.13\%) and achieved $1.8 \%$ in average. The genus Psychrilyobacter was also rare and accounted for only $0.04 \%$ in the control group, while the selective pressure of ciprofloxacin (Group Q) could stimulate this genus and the abundance reached the maximum $(25.48 \%)$ on Day 5. Although this genus then decreased again as the incubation time prolonging, the abundance still accounted for $1.59 \%$ in the sample Q4. Similarly, the selective pressure of ampicillin sodium (Group R) made the genus Sphaerochaeta and an unclassified genus of family Peptostreptococcaceae became abundant, which accounted for $12.56 \%$ and $5.41 \%$ in average in the ampicillin sodium-treated group (Group R), respectively. In the tylosin-treated group (Group T), a genus from order Clostridiales changed dramatically. The abundance of this genus first decreased from $0.01 \%$ to $0 \%$ during first 5 days and then increased to $30.16 \%$ on Day 21 . Under the condition of second addition of tylosin, the abundance even reached the maximum $(45.85 \%)$. The addition of antibiotics changed the structure and abundance of microbial community greatly. Moreover, 29 genera were not detected in the control sample A0 but could be detected in at least one antibiotic-treated group. For example, the genus Fusobacterium could not be detected in the control sample A0, but after enrichment culturing, the abundance increased to $3.87 \%-14.12 \%$ in average. In the zinc bacitracin-treated group (Group P), its abundance was 3.54\%-14.73\%, especially the second addition of zinc bacitracin made the abundance of genus Fusobacterium increased to $12.69 \%$ in the sample JP4. In the tylosin-treated group (Group T), the abundance of genus Fusobacterium increased to $2.58 \%$ when applying selective pressure for 5 days, then its abundance became very low even could not be detected in the sample JT4. The genus Proteiniphilum was only detected in the tylosin-treated group (Group T) and chloromycetin-treated group (Group S), with an average abundance of $6.41 \%$ and $0.02 \%$, respectively. The genera Peptostreptococcus and Macellibacteroides were also not detected in the control group but could be detected under the selective pressure of tylosin (The data of this part were not shown in the paper).

Conversely, some genera existing in the control group even in high abundance, could be inhibited by the antibiotic and could not be detected under the pressure of antibiotic. The genus Dethiosulfatibacter had the average abundance of $3.64 \%$ in the control group, but the pressure of ampicillin sodium (Group R) inhibited this genus greatly and made this genus cannot be detected in all the ampicillin sodium-treated samples. Genus Marinifilum had the average abundance of $6.24 \%$ in the control group, but the pressure of tylosin made this genus could not be detected in all the tylosin-treated samples (Table S3).

\subsubsection{Comparative Analysis of Microbial Community Structures under Different Antibiotic} Selective Pressures

The variance analysis of the microbial community compositions among different samples was evaluated using Hierarchical clustering analysis and nonmetric multidimensional scaling (NMDS) analysis at OTU level. Figure S3 exhibited the Hierarchical clustering tree at OTU level. The control sample A0 formed a single branch, suggesting that the microbial community composition of A0 was significantly different from other samples. In general, the samples of the same treatment group gathered together, being separate from the other branches formed by other groups. This illustrated that 
different type of antibiotics affected the structure of microbial community individually, endowing each group with a special microbial community (Figure S3). In particular, there were also some samples that belonged to different groups having similar microbial community composition and formed one linkage. For example, the samples A1 and P1 and the samples S1 and R1, which were the samples of the same incubation time (Figure S3). This may be due to the selective pressure had not yet maintained for enough time and the microbial community composition of these four samples was influenced more significantly by the enrichment culturing time than by the antibiotic.

NMDS analysis based on the Abund-Jaccard distance were conducted at OTU level (Figure S4). In general, the samples from the same treatment group closely clustered with a few exceptions. For instance, the samples A0, S1 and Q1 were not closely clustered within their relative groups. The samples from different antibiotic treatments had a significant difference in microbial community compositions and clustered separately. Nonmetric multidimensional scaling and pairwise analyses of similarities (ANOSIM) analysis showed that the microbial community compositions of the different antibiotic-treated groups and the control group were statistically significantly different $(P=0.001)$. In addition, the samples from tylosin-treated group (Group T) were far from the other groups, indicating the selective pressure of tylosin made the microbial community compositions more different. SIMPER analysis was performed based on OTUs to identify the dissimilarity contribution of each taxon. The main results are shown in Table S4. Taxa were considered among the most discriminating species if their contribution to percentage dissimilarity was $\geq 5 \%$. Two species of family Desulfobacteraceae genus Desulfobacter (OTU2543 and OTU1450) were the most common contributive taxa to the dissimilarity of bacterial community between antibiotic-treated groups and the control group. Between Group A and P, Q, R, U, Desulfobacter sp. (OTU2543) was the most important dissimilarity contributor, and the contribution percentage was $12.88 \%, 11.65 \%, 8.6 \%$ and $14.03 \%$, respectively. Comparing Group A with S, the most important dissimilarity contributor was an unclassified group of phylum Firmicutes order Clostridiales (OTU1295, 10.45\%). Comparing Group A with T, the most important dissimilarity contributor was also an unclassified Firmicutes of order Clostridiales (OTU2556, 14.39\%).

The difference of enrichment culturing time also resulted in the separation of the samples from the same treatment, for example $\mathrm{T} 1$ and P1. Comparing the effect of different enrichment time on the microbial community compositions, ANOSIM analysis suggested that the duration time of antibiotic exposure also affect the microbial community composition statistically significantly $(P=0.009)$. SIMPER analysis (Table S5) showed that Vibrio sp. (OTU2034) was the most important contributive species to the dissimilarity of bacterial community after enrichment culturing for 5 days. Desulfobacter sp. (OTU2543) was the most important contributor to dissimilarity of bacterial community between control sample and the samples incubating for longer time.

The differences between antibiotic-treated groups were further analyzed based on phyla level and genus level (Figures S5 and S6). From Figure S5, we knew that the abundance of phyla Bacteroidetes, Firmicutes, Spirochetae, Tenericutes, Synergistetes, Cloacimonetes and Lentisphaerae were significantly different and caused significant statistical differences between different treatments. At the genus level (Figure S6), Marinifilum, Fusobacterium, Dethiosulfatibacter, Psychrilyobacter, Sunxiuqinia, Draconibacterium and three unclassified genera, were the main genera causing statistical differences between different treatments. Apart from the influence of the antibiotics, the duration time also had effect on the microbial community. Combining the samples of the same enrichment time as a group, the differences between groups of different time were also analyzed at both phylum level and genus level (Figures S7 and S8). The phyla of Firmicutes, Fusobacteria, Spirochetae, Synergistetes and Cloacimonetes were the main factors resulting in the significant difference in the microbial community between the samples experiencing antibiotic exposure for different time (Figure S7). At the genus level, Desulfobacter, Marinifilum, Vibrio, Fusobacterium, Propionigenium, Sphaerochaeta, Desulfovibrio, Dethiosulfatibacter, Sunxiuqinia and an unclassified genus, changed significantly as the time prolonging and caused statistical difference in the microbial community between the samples experiencing antibiotic exposure for different time (Figure 
S8). Hence, both the antibiotic and different treatment time could affect the microbial community of the sediment samples significantly.

The high-throughput $16 \mathrm{~S}$ rRNA gene amplicon sequencing results showed that the 2822 OTUs in all the samples were annotated to 49 phyla, 103 class, 220 orders, 347 families, 624 genera and 1122 species, suggesting the richness and diversity of the marine sediment. However, the cultivable bacteria were only of 4 phyla (Actinobacteria, Bacteroidetes, Firmicutes and Proteobacteria), indicating there were large number of bacteria that could not be isolated and cultured under the existing conditions. The abundance of Actinobacteria was not very high, but this phylum could be cultivated in most of the samples. This may be due to that Actinomycetes have great resistance to antibiotics. Under the selective pressure of antibiotics, the composition of the microbial community changed and still a large number of OTUs could be clustered and many strains could be isolated, illustrating the seriousness of bacterial resistance. However, optimistically, some potential novel isolates were obtained upon addition of antibiotics, providing a new method for cultivation and isolation of novel bacteria.

\subsection{Analysis of Bacterial Functions}

PICRUSt was used to predict the bacterial potential functions in the samples of different antibiotic-treated groups. The output of PICRUSt consists of functional gene abundance known as KOs (Kyoto Encyclopedia of Genes and Genomes (KEGG) Orthologs (KOs)). At the top level of pathways modules, metabolism $(24.91 \%-27.37 \%)$, genetic information processing (GIP, $15.00 \%-18.03 \%)$, environmental information processing (EIP, $11.92 \%-12.41 \%)$ and cellular processes $(\mathrm{CP}, 1.96 \%-5.44 \%)$ were the major functional categories, followed by human diseases $(0.69 \%-1.12 \%)$ and organismal systems $(0.57 \%-0.83 \%)$. It is worth mentioning that the bacterial functions belonged to "Unclassified" accounted for $14.52 \%-15.39 \%$, suggesting that further studies are needed to clarify the new functions.

As shown in Figure 9, within different antibiotic-treated groups (Figure 9a) or within different enrichment time groups (Figure $9 \mathrm{~b}$ ), the average relative abundance of pathways modules which at the top level were the same, i.e., metabolism $(42.47 \%-44.50 \%)$, genetic information processing (GIP, $18.53 \%-19.10 \%$ ), environmental information processing (EIP, $15.14 \%-16.34 \%$ ) and cellular processes (CP, 5.20\%-5.98\%). The average relative abundance of human diseases and organismal systems in different antibiotic-treated groups was lower than $1 \%$. It is worth mentioning that the bacterial functions belonging to "Unclassified" accounted for $15.08 \%-15.26 \%$, suggesting that further studies are needed to illuminate the new functions. In Figure $9 b$, the samples of the same enrichment time were combined as one group, the main functions were also metabolism, GIP, EIP and CP.

To further compare different samples, the top-50 bacterial functions at functional subcategory (level three) were investigated (Figure 10). Transporters was the most abundant pathway under EIP in all the samples, followed by two-component system were the main pathways under EIP in each sample, which showed higher relative abundance in tylosin-treated group and the relative abundance of bacterial motility proteins and bacterial chemotaxis were the main pathways under CP. DNA repair and recombination proteins, ribosome and chromosome were the main pathways under GIP. ABC transporters and secretion systems were the main pathways under EIP. 


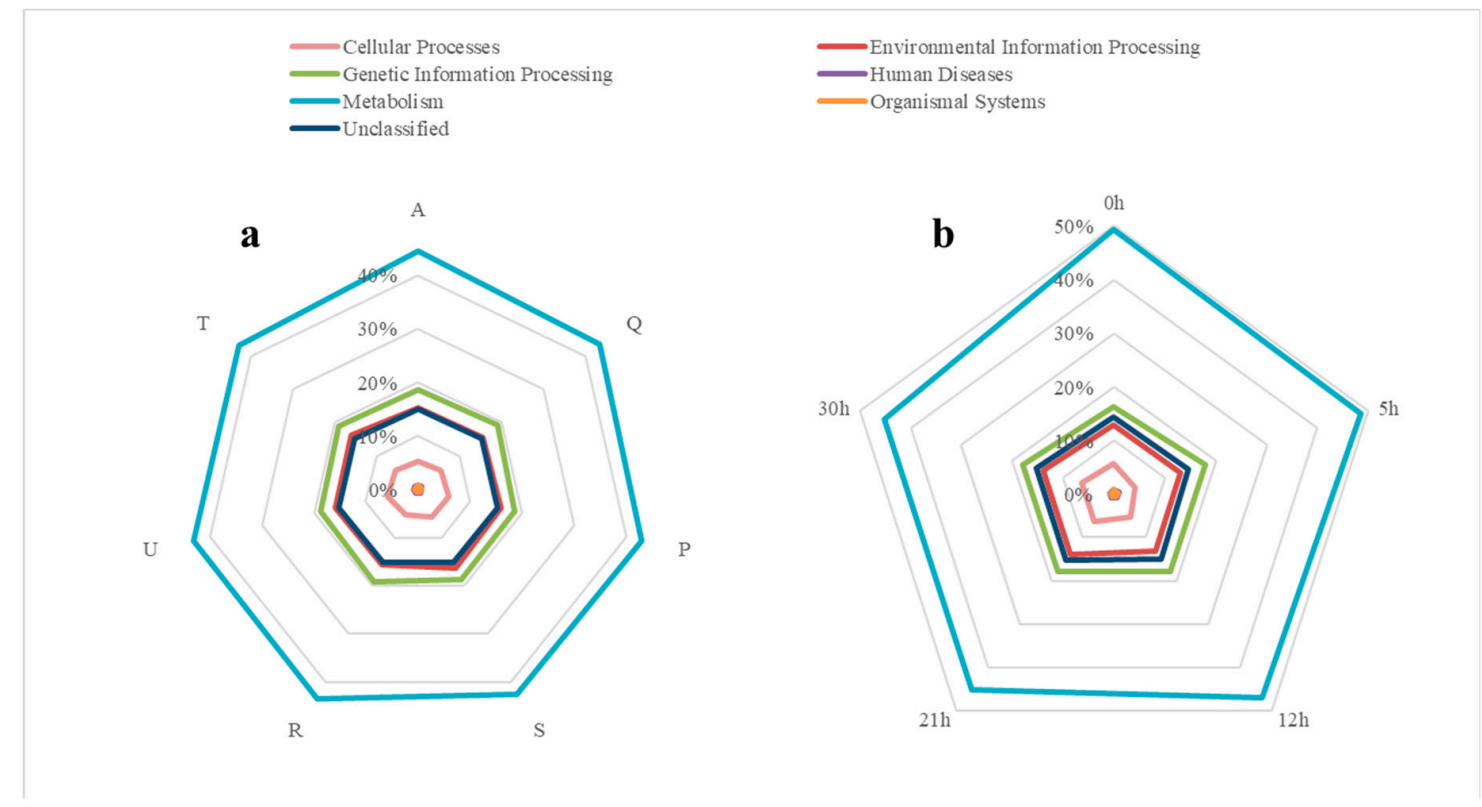

Figure 9. Bacterial functions analysis. (a) Distributions of bacterial functions at the top level of pathways modules in different antibiotic-treated groups; (b) distributions of bacterial functions at the top level of pathways modules in the groups of different enrichment time. GIP-genetic information processing; $\mathrm{EIP}$ - environmental information processing; $\mathrm{CP}$-cellular processes.

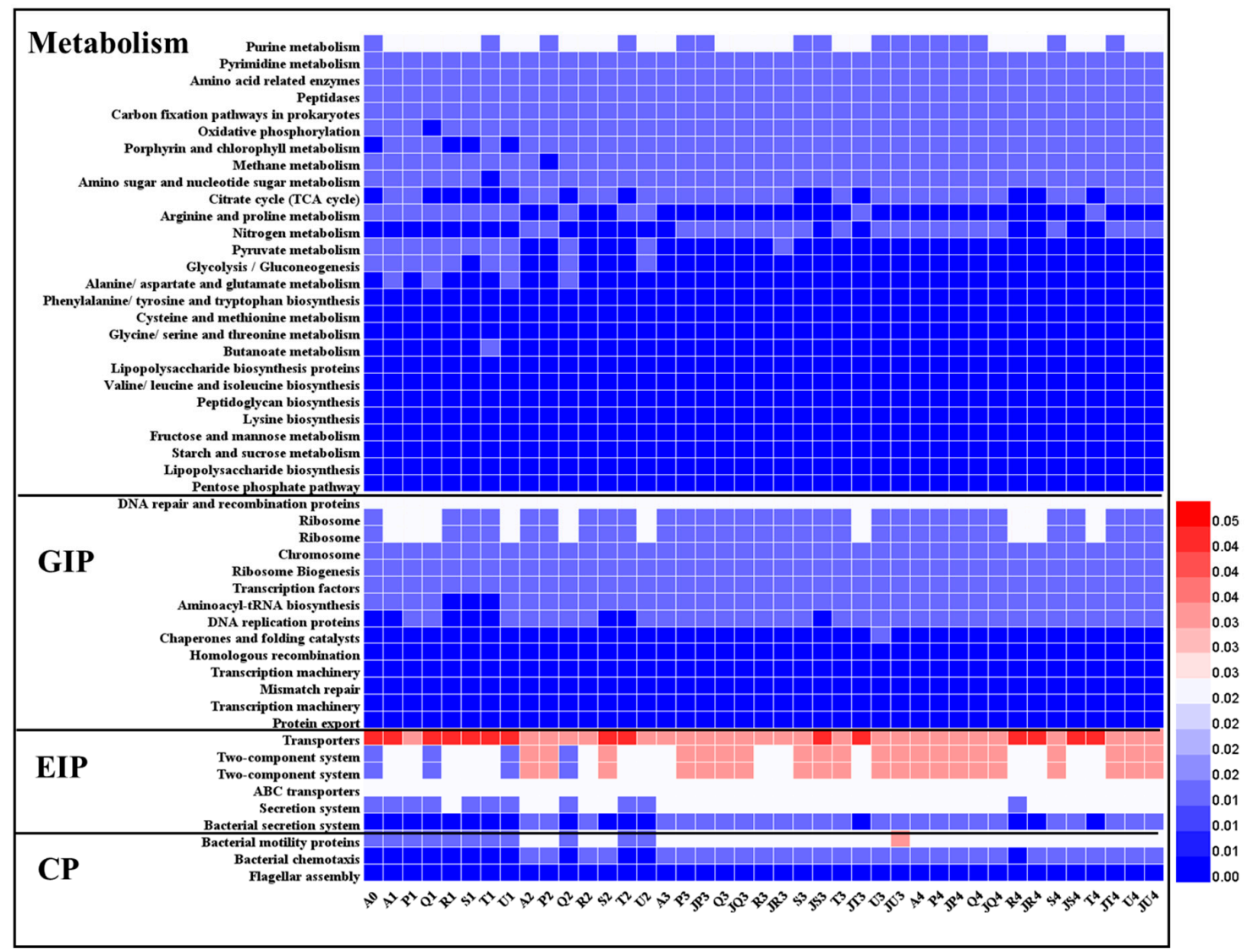

Figure 10. Relative abundance of top-50 bacterial functions at functional subcategory (level three). GIP-genetic information processing; EIP-environmental information processing; $\mathrm{CP}$-cellular processes. 


\subsection{Antibiotic Resistance Genes Analysis}

The shifts in bacterial community composition are correlated with antibiotic resistance genes (ARGs) [50]. The occurrence of predicted ARGs in the samples of different antibiotic-treated groups was investigated. A total of 30 ARGs were detected in the samples. The predicted ARGs were mainly confer resistance to cationic antimicrobial peptide (CAMPs, $18.63 \%$ ), followed by macrolide $(5.70 \%)$, aminoglycoside $(5.07 \%)$, fluoroquinolone $(4.58 \%)$, imipenem $(3.47 \%)$ and vancomycin $(0.92 \%)$ (Figure 11). Cationic antimicrobial peptides (CAMPs) are present in the innate host defense system and play an important role to against microbial infections and microbial products [51]. Bacteria constantly encounter CAMPs during infection, so they have developed some mechanisms to confer intrinsic or inducible resistance, which may result in the highest abundance of ARGs for CAMPs. In addition, there were still $51.88 \%$ of ARGs conferring resistance to efflux antibiotics. Efflux pumps were identified as the most common resistance mechanisms in many environment, such as estuary and deep ocean sediments [52] and activated sludge samples [53], which were consistent with our study. Heatmap analysis of ARGs subtypes was further conducted (Figure 11). Among the genes that encode for CAMPs, amiA/amiC and $\operatorname{degP} / \mathrm{dsbA}$ were the most abundant subtypes. For beta_lactam, ampC was more abundant than bla. There were 10 ARGs subtypes conferring resistance to other antibiotics and the gene counts of acrAD-tolC, acrAB-tolC/smeDEF, ameABC, mexAB-oprM and mdtABC were higher than the other ARGs subtypes in most of samples. One ARGs type for imipenem was obtained, i.e., oprD. Comparing the different samples, it could be found that the samples that incubating for 5 days in each antibiotic-treated group, i.e., A1, P1, R1, S1, T1 and U1, appear to be fairly uniform and were different from the other samples. The samples had the more abundant ARGs than that of the other samples. Overall, ARGs existed in all the groups and the dominant subtypes were similar in different antibiotic-treated groups. Moreover, their distributions were affected by enrichment time.

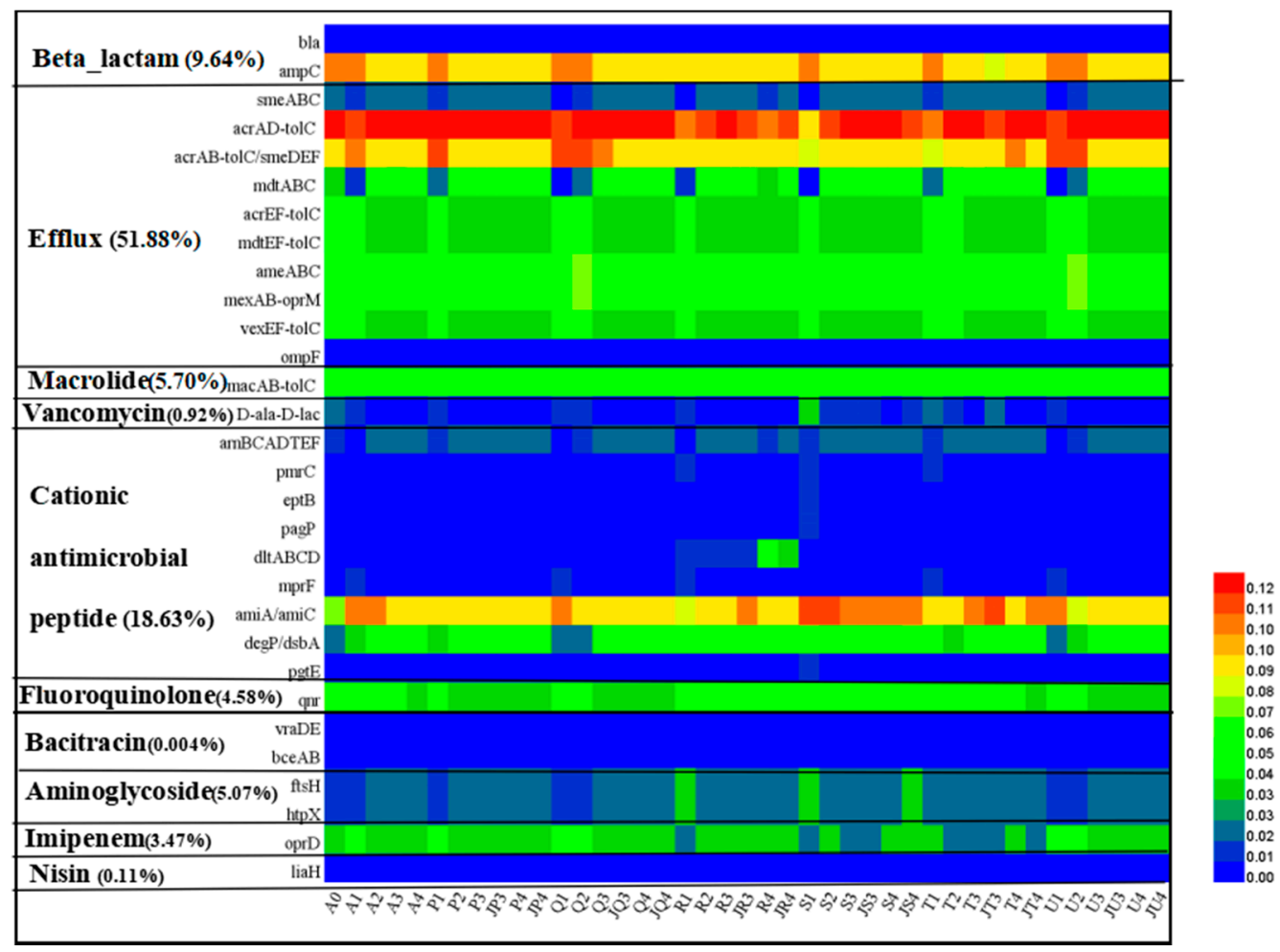

Figure 11. Heatmap analysis of antibiotic resistance genes (ARGs) in all the samples. Percentages in parentheses represent the distributions of antibiotics to which these ARGs confer resistance. 


\section{Conclusions}

Although some studies have already reported the effects of antibiotics on the bacterial communities in different environments, this is one of the few studies to focus on the marine environment, especially the mariculture sediments. In addition, dynamic changes of bacterial communities under the antibiotic selective pressure for different enrichment culturing time was investigated. Based on not only classical culture-dependent method, but also culture-independent microbial diversity analysis by 16S rRNA gene sequencing, we avoided the bias obtained when evaluating the microbial community change based only on cultivable bacteria. In conclusion, the findings in this paper revealed that antibiotic selective pressures could significantly affect the structure and abundance of microbial community. Bacteroidetes, Firmicutes and Proteobacteria were the dominant phyla in the sediment of sea cucumber pond. Simultaneously, antibiotic treatments could promote some microbial groups and improve their abundance. This may provide a potential new method for the isolation and culture of some potential novel strains. Exposure to antibiotics for different time also could lead to the change in the composition and abundance of the microbial community. Metabolism was the predominant bacterial function. A total of 30 ARGs were detected in the samples. The dominant subtypes were similar in different antibiotic-treated groups, and their distributions was affected by enrichment time. Based on above results, continued research is necessary to evaluate the resistance gene clusters in related bacteria induced by antibiotic exposure and their potential risk of genetic transfer in the environment.

Supplementary Materials: The following are available online at http://www.mdpi.com/2077-1312/8/8/604/s1, Table S1: The information of antibiotics used in this study, Table S2: The dominant phyla (average abundance > $1.0 \%$ ) in each treatment, Table S3: The dominant genera (average abundance $>1.0 \%$ ) in each treatment, Table S4: SIMPER analysis of the bacterial community in antibiotic-treated group and Control group at the OTU level, Table S5: SIMPER analysis of the bacterial community in groups of different enrichment time at the OTU level, Figure S1: The number of shared and unique OTUs in the samples of the same group. (a) zinc bacitracin-treated group; (b) ciprofloxacin-treated group; (c) ampicillin sodium-treated group; (d) chloramphenicol-treated group; (e) tylosin-treated group; (f) tetracycline-treated group, Figure S2: Composition of microbial community at the class level, Figure S3: Hierarchical clustering tree at OTU level, Figure S4: NMDS (Abund-Jaccard distance) plot based on OTUs abundance showing the microbial composition variations among different samples, Figure S5: Comparison of control group and different antibiotic-treated groups at phylum level, Figure S6: Comparison of control group and different antibiotic-treated groups at genus level, Figure S7: Comparison of samples of different enrichment time at phylum level, Figure S8: Comparison of samples of different enrichment time at genus level.

Author Contributions: Z.-J.D. and M.-Q.Y. contributed conception and design of the study; M.-Q.Y. performed the experiments, made analysis of the data and prepared the original draft; Z.-J.D. and G.-J.C. reviewed and edited the draft. All authors contributed to manuscript revision, read and approved the submitted version. All authors have read and agreed to the published version of the manuscript.

Funding: This work was funded by the National Natural Science Foundation of China (31770002).

Conflicts of Interest: The authors declare no conflicts of interest.

\section{References}

1. Sharma, V.K.; Johnson, N.; Cizmas, L.; McDonald, T.J.; Kim, H. A review of the influence of treatment strategies on antibiotic resistant bacteria and antibiotic resistance genes. Chemosphere 2016, 150, 702-714. [CrossRef] [PubMed]

2. Zhu, S.; Chen, H.; Li, J. Sources, distribution and potential risks of pharmaceuticals and personal care products in Qingshan Lake basin, Eastern China. Ecotoxicol. Environ. Saf. 2013, 96, 154-159. [CrossRef] [PubMed]

3. Stockwell, V.O.; Duffy, B. Use of antibiotics in plant agriculture. Rev. Sci. Tech. 2012, 31, 199-210. [CrossRef] [PubMed]

4. Cabello, F.C. Heavy use of prophylactic antibiotics in aquaculture: A growing problem for human and animal health and for the environment. Environ. Microbiol. 2006, 8, 1137-1144. [CrossRef] [PubMed]

5. Liu, X.; Lu, S.; Guo, W.; Xi, B.; Wang, W. Antibiotics in the aquatic environments: A review of lakes, China. Sci. Total Environ. 2018, 627, 1195-1208. [CrossRef] 
6. Klein, E.Y.; Van Boeckel, T.P.; Martinez, E.M.; Pant, S.; Gandra, S.; Levin, S.A.; Goossens, H.; Laxminarayan, R. Global increase and geographic convergence in antibiotic consumption between 2000 and 2015. Proc. Natl. Acad. Sci. USA 2018, 115, E3463-E3470. [CrossRef]

7. Zhou, L.J.; Ying, G.G.; Liu, S.; Zhao, J.L.; Yang, B.; Chen, Z.F.; Lai, H.J. Occurrence and fate of eleven classes of antibiotics in two typical wastewater treatment plants in South China. Sci. Total Environ. 2013, 452, 365-376. [CrossRef]

8. Dolliver, H.; Gupta, S. Antibiotic Losses in Leaching and Surface Runoff from Manure-Amended Agricultural Land. J. Environ. Qual. 2008, 37, 1227. [CrossRef]

9. Hanna, N.; Sun, P.; Sun, Q.; Li, X.; Yang, X.; Ji, X.; Zou, H.; Ottoson, J.; Nilsson, L.E.; Berglund, B.; et al. Presence of antibiotic residues in various environmental compartments of Shandong province in eastern China: Its potential for resistance development and ecological and human risk. Environ. Int. 2018, 114, 131-142. [CrossRef]

10. Andremont, A.; Walsh, T.R. The role of sanitation in the development and spread of antimicrobial resistance. AMR Control 2015, 67, 68-73.

11. Qiao, M.; Ying, G.; Singer, A.C.; Zhu, Y. Review of antibiotic resistance in China and its environment. Environ. Int. 2018, 110, 160-172. [CrossRef] [PubMed]

12. Guo, X.; Yang, Y.; Lu, D.; Niu, Z.; Feng, J.; Chen, Y.; Tou, F.; Garner, E.; Xu, J.; Liu, M.; et al. Biofilms as a sink for antibiotic resistance genes (ARGs) in the Yangtze Estuary. Water Res. 2018, 129, 277-286.

13. Allen, H.K.; Donato, J.; Wang, H.H.; Cloud-Hansen, K.A.; Davies, J.; Handelsman, J. Call of the wild: Antibiotic resistance genes in natural environments. Nat. Rev. Microbiol. 2010, 8, 251-259. [CrossRef]

14. Näslund, J.; Hedman, J.E.; Agestrand, C. Effects of the antibiotic ciprofloxacin on the bacterial community structure and degradation of pyrene in marine sediment. Aquat. Toxicol. 2008, 90, 223-227. [CrossRef]

15. Guan, Y.; Jia, J.; Wu, L.; Xue, X.; Zhang, G.; Wang, Z. Analysis of bacterial community characteristics, abundance of antibiotics and antibiotic resistance genes along a pollution gradient of Ba river in Xi'an, China. Front. Microbiol. 2018, 9, 3191. [CrossRef]

16. Lu, T.; Zhu, Y.; Ke, M.; Peijnenburg, W.J.G.M.; Zhang, M.; Wang, T.; Chen, J.; Qian, H. Evaluation of the taxonomic and functional variation of freshwater plankton communities induced by trace amounts of the antibiotic ciprofloxacin. Environ. Int. 2019, 126, 268-278. [CrossRef]

17. Semedo, M.; Song, B.; Sparrer, T.; Phillips, R.L. Antibiotic effects on microbial communities responsible for denitrification and $\mathrm{N}_{2} \mathrm{O}$ production in grassland soils. Front. Microbiol. 2018, 9, 2121. [CrossRef]

18. Costanzo, S.D.; Murby, J.; Bates, J. Ecosystem response to antibiotics entering the aquatic environment. Mar. Pollut. Bull. 2005, 51, 218-223. [CrossRef]

19. Thiele-Bruhn, S.; Beck, I. Effects of sulfonamide and tetracycline antibiotics on soil microbial activity and microbial biomass. Chemosphere 2005, 59, 457-465. [CrossRef]

20. Bell, T.; Newman, J.A.; Silverman, B.W.; Turner, S.L.; Lilley, A.K. The contribution of species richness and composition to bacterial services. Nature 2005, 436, 1157-1160. [CrossRef]

21. Cabello, F.C.; Godfrey, H.P.; Buschmann, A.H.; Dolz, H.J. Aquaculture as yet another environmental gateway to the development and globalisation of antimicrobial resistance. Lancet Infect. Dis. 2016, 16, e127-e133. [CrossRef]

22. Henriksson, P.J.G.; Rico, A.; Troell, M.; Klinger, D.H.; Buschmann, A.H.; Saksida, S.; Chadag, M.V.; Zhang, W. Unpacking factors influencing antimicrobial use in global aquaculture and their implication for management: A review from a systems perspective. Sustain. Sci. 2018, 13, 1105-1120. [CrossRef] [PubMed]

23. Rico, A.; Satapornvanit, K.; Haque, M.M.; Min, J.; Nguyen, P.T.; Telfer, T.C.; van den Brink, P.J. Use of chemicals and biological products in Asian aquaculture and their potential environmental risks: A critical review. Rev. Aquac. 2012, 4, 75-93. [CrossRef]

24. Deng, Y.; Wu, Y.; Tan, A.; Huang, Y.; Jiang, L.; Xue, H.; Wang, W.; Luo, L.; Zhao, F. Analysis of Antimicrobial Resistance Genes in Aeromonas spp. Isolated from Cultured Freshwater Animals in China. Microb. Drug Resist. 2014, 20, 350-356. [CrossRef]

25. Aedo, S.; Ivanova, L.; Tomova, A.; Cabello, F.C. Plasmid-Related Quinolone Resistance Determinants in Epidemic Vibrio parahaemolyticus, Uropathogenic Escherichia coli, and Marine Bacteria from an Aquaculture Area in Chile. Microb. Ecol. 2014, 68, 324-328. [CrossRef] 
26. Di Cesare, A.; Luna, G.M.; Vignaroli, C.; Pasquaroli, S.; Tota, S.; Paroncini, P.; Biavasco, F. Aquaculture can promote the presence and spread of antibiotic-resistant Enterococci in marine sediments. PLoS ONE 2013, 8 , e62838. [CrossRef]

27. Ma, J.; Zhu, D.; Sheng, G.D.; O'Connor, P.; Zhu, Y. Soil oxytetracycline exposure alters the microbial community and enhances the abundance of antibiotic resistance genes in the gut of Enchytraeus crypticus. Sci. Total Environ. 2019, 673, 357-366. [CrossRef]

28. Shi, L.; Ge, B.; Liu, B.; Liu, X.; Jiang, M.; Zhang, K. Impact of Wuyiencin application on the soil microbial community and fate of typical antibiotic resistance genes. Sci. Rep. 2019, 9, 4016. [CrossRef]

29. Huerta, B.; Marti, E.; Gros, M.; López, P.; Pompêo, M.; Armengol, J.; Barceló, D.; Balcázar, J.L.; Rodríguez-Mozaz, S.; Marcé, R. Exploring the links between antibiotic occurrence, antibiotic resistance, and bacterial communities in water supply reservoirs. Sci. Total Environ. 2013, 456, 161-170. [CrossRef]

30. Zhao, R.; Feng, J.; Liu, J.; Fu, W.; Li, X.; Li, B. Deciphering of microbial community and antibiotic resistance genes in activated sludge reactors under high selective pressure of different antibiotics. Water Res. 2019, 151, 388-402.

31. Zhang, Y.; Geng, J.; Ma, H.; Ren, H.; Xu, K.; Ding, L. Characterization of microbial community and antibiotic resistance genes in activated sludge under tetracycline and sulfamethoxazole selection pressure. Sci. Total Environ. 2016, 571, 479-486. [CrossRef] [PubMed]

32. Liu, H.; Yang, Y.; Sun, H.; Zhao, L.; Liu, Y. Effect of tetracycline on microbial community structure associated with enhanced biological N\&P removal in sequencing batch reactor. Bioresour. Technol. 2018, 256, 414-420. [PubMed]

33. Zou, S.; Xu, W.; Zhang, R.; Tang, J.; Chen, Y.; Zhang, G. Occurrence and distribution of antibiotics in coastal water of the Bohai Bay, China: Impacts of river discharge and aquaculture activities. Environ. Pollut. 2011, 159, 2913-2920. [CrossRef]

34. Xie, H.; Wang, X.; Chen, J.; Li, X.; Jia, G.; Zou, Y.; Zhang, Y.; Cui, Y. Occurrence, distribution and ecological risks of antibiotics and pesticides in coastal waters around Liaodong Peninsula, China. Sci. Total Environ. 2019, 656, 946-951. [CrossRef] [PubMed]

35. Du, J.; Zhao, H.; Liu, S.; Xie, H.; Wang, Y.; Chen, J. Antibiotics in the coastal water of the South Yellow Sea in China: Occurrence, distribution and ecological risks. Sci. Total Environ. 2017, 595, 521-527. [CrossRef] [PubMed]

36. Zhang, R.; Tang, J.; Li, J.; Zheng, Q.; Liu, D.; Chen, Y.; Zou, Y.; Chen, X.; Luo, C.; Zhang, G. Antibiotics in the offshore waters of the Bohai Sea and the Yellow Sea in China: Occurrence, distribution and ecological risks. Environ. Pollut. 2013, 174, 71-77. [CrossRef]

37. Turnidge, J.; Christiansen, K. Antibiotic use and resistance-Proving the obvious. Lancet 2005, 365, 548-549. [CrossRef]

38. Mu, D.S.; Liang, Q.Y.; Wang, X.M.; Lu, D.C.; Shi, M.J.; Chen, G.J.; Du, Z.J. Metatranscriptomic and comparative genomic insights into resuscitation mechanisms during enrichment culturing. Microbiome 2018, 6, 230. [CrossRef]

39. Li, X.; Xiao, H.; Zhang, W.; Li, Y.; Tang, X.; Duan, J.; Yang, Z.; Wang, J.; Guan, F.; Ding, G. Analysis of cultivable aerobic bacterial community composition and screening for facultative sulfate-reducing bacteria in marine corrosive steel. J. Oceanol. Limnol. 2019, 37, 600-614. [CrossRef]

40. Liu, Q.Q.; Wang, Y.; Li, J.; Du, Z.J.; Chen, G.J. Saccharicrinis carchari sp. nov., isolated from a shark, and emended descriptions of the genus Saccharicrinis and Saccharicrinis fermentans. Int. J. Syst. Evol. Microbiol. 2014, 64, 2204-2209. [CrossRef]

41. Song, C.; Wang, B.; Tan, J.; Zhu, L.; Lou, D.; Cen, X. Comparative analysis of the gut microbiota of black bears in China using high-throughput sequencing. Mol. Genet. Genom. 2017, 292, 407-414. [CrossRef] [PubMed]

42. Edgar, R.C. UPARSE: Highly accurate OTU sequences from microbial amplicon reads. Nat. Methods 2013, 10, 996-998. [CrossRef] [PubMed]

43. Caporaso, J.G.; Kuczynski, J.; Stombaugh, J.; Bittinger, K.; Bushman, F.D.; Costello, E.K.; Fierer, N.; Pena, A.G.; Goodrich, J.K.; Gordon, J.I.; et al. QIIME allows analysis of high-throughput community sequencing data. Nat. Methods 2010, 7, 335-336. [CrossRef] [PubMed]

44. Quast, C.; Pruesse, E.; Yilmaz, P.; Gerken, J.; Schweer, T.; Yarza, P.; Peplies, J.; Glockner, F.O. The SILVA ribosomal RNA gene database project: Improved data processing and web-based tools. Nucleic Acids Res. 2013, 41, D590-D596. [CrossRef] [PubMed] 
45. Langille, M.G.I.; Zaneveld, J.; Caporaso, J.G.; McDonald, D.; Knights, D.; Reyes, J.A.; Clemente, J.C.; Burkepile, D.E.; Vega Thurber, R.L.; Knight, R.; et al. Predictive functional profiling of microbial communities using 16S rRNA marker gene sequences. Nat. Biotechnol. 2013, 31, 814-821. [CrossRef]

46. Zhao, Z.; Wang, J.; Han, Y.; Chen, J.; Liu, G.; Lu, H.; Yan, B.; Chen, S. Nutrients, heavy metals and microbial communities co-driven distribution of antibiotic resistance genes in adjacent environment of mariculture. Environ. Pollut. 2017, 220, 909-918. [CrossRef]

47. Wang, S.; Cui, Y.; Li, A.; Wang, D.; Zhang, W.; Chen, Z. Seasonal dynamics of bacterial communities associated with antibiotic removal and sludge stabilization in three different sludge treatment wetlands. J. Environ. Manag. 2019, 240, 231-237. [CrossRef]

48. Xu, Z.; Jiang, Y.; Te, S.; He, Y.; Gin, K. The Effects of Antibiotics on Microbial Community Composition in an Estuary Reservoir during Spring and Summer Seasons. Water 2018, 10, 154. [CrossRef]

49. Zhang, M.; Yuan, L.; Li, Z.; Zhang, H.; Sheng, G. Tetracycline exposure shifted microbial communities and enriched antibiotic resistance genes in the aerobic granular sludge. Environ. Int. 2019, 130, 104902. [CrossRef]

50. Zhang, H.; He, H.; Chen, S.; Huang, T.; Lu, K.; Zhang, Z.; Wang, R.; Zhang, X.; Li, H. Abundance of antibiotic resistance genes and their association with bacterial communities in activated sludge of wastewater treatment plants: Geographical distribution and network analysis. J. Environ. Sci. China 2019, 82, 24-38. [CrossRef]

51. Tzeng, Y.; Ambrose, K.D.; Zughaier, S.M.; Zhou, X.; Miller, Y.K.; Shafer, W.M.; Stephens, D.S. Cationic Antimicrobial Peptide Resistance in Neisseria meningitidis. J. Bacteriol. 2005, 187, 5387-5396. [CrossRef] [PubMed]

52. Chen, B.; Yang, Y.; Liang, X.; Yu, K.; Zhang, T.; Li, X. Metagenomic Profiles of Antibiotic Resistance Genes (ARGs) between Human Impacted Estuary and Deep Ocean Sediments. Environ. Sci. Technol. 2013, 47, 12753-12760. [CrossRef] [PubMed]

53. Zhang, T.; Yang, Y.; Pruden, A. Effect of temperature on removal of antibiotic resistance genes by anaerobic digestion of activated sludge revealed by metagenomic approach. Appl. Microbiol. Biotechnol. 2015, 99, 7771-7779. [CrossRef] [PubMed]

(C) 2020 by the authors. Licensee MDPI, Basel, Switzerland. This article is an open access article distributed under the terms and conditions of the Creative Commons Attribution (CC BY) license (http://creativecommons.org/licenses/by/4.0/). 